\title{
Intestinal microbiota influences clinical outcome and side effects of early breast cancer treatment
}

\author{
Safae Terrisse ${ }^{1,2,3,4} \cdot$ Lisa Derosa $^{1,2,5} \cdot$ Valerio lebba $^{1,2} \cdot$ François Ghiringhelli $\mathbb{D}^{6,7,8,9} \cdot$ Ines Vaz-Luis $^{10,11}$. \\ Guido Kroemer $\mathbb{D}^{12,13,14,15} \cdot$ Marine Fidelle $\mathrm{e}^{1,2,3,5} \cdot$ Stergios Christodoulidis $\mathbb{D}^{16,17}$. Nicola Segata $\mathbb{D}^{18,19}$. \\ Andrew Maltez Thomas $\mathbb{D}^{19}$. Anne-Laure Martin ${ }^{20}$ - Aude Sirven ${ }^{20} \cdot$ Sibille Everhard $^{20} \cdot$ Fanny Aprahamian $^{13}$.

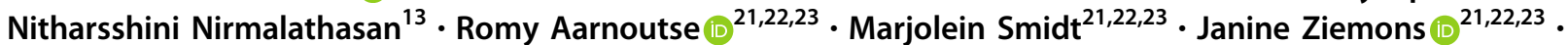 \\ Carlos Caldas $\mathbb{D}^{24}$. Sibylle Loibl ${ }^{25,26}$. Carsten Denkert ${ }^{27}$. Sylvere Durand ${ }^{13}$. Claudia Iglesias $^{13}$. \\ Filippo Pietrantonio ${ }^{28} \cdot$ Bertrand Routy $^{29} \cdot$ Fabrice André $^{1,10,11,16} \cdot$ Edoardo Pasolli $^{30,31}$. Suzette Delaloge ${ }^{1,11}$. \\ Laurence Zitvogel $\mathbb{1}^{1,2,3,5}$
}

Received: 18 January 2021 / Revised: 5 April 2021 / Accepted: 12 April 2021 / Published online: 7 May 2021

(c) The Author(s) 2021. This article is published with open access

\begin{abstract}
The prognosis of early breast cancer (BC) relies on cell autonomous and immune parameters. The impact of the intestinal microbiome on clinical outcome has not yet been evaluated. Shotgun metagenomics was used to determine the composition of the fecal microbiota in 121 specimens from 76 early BC patients, 45 of whom were paired before and after chemotherapy. These patients were enrolled in the CANTO prospective study designed to record the side effects associated with the clinical management of BC. We analyzed associations between baseline or post-chemotherapy fecal microbiota and plasma metabolomics with $\mathrm{BC}$ prognosis, as well as with therapy-induced side effects. We examined the clinical relevance of these findings in immunocompetent mice colonized with $\mathrm{BC}$ patient microbiota that were subsequently challenged with histo-compatible mouse $\mathrm{BC}$ and chemotherapy. We conclude that specific gut commensals that are overabundant in BC patients compared with healthy individuals negatively impact BC prognosis, are modulated by chemotherapy, and may influence weight gain and neurological side effects of $\mathrm{BC}$ therapies. These findings obtained in adjuvant and neoadjuvant settings warrant prospective validation.
\end{abstract}

\section{Introduction}

Breast cancer (BC) ranks first in women, and is the second cause of death in this gender. In addition to ageing and genetics, environmental factors (diet, ethanol consumption, endocrine disruptors, sedentary lifestyle) may contribute to the development of the disease. Several mechanisms might account for the putative impact of the microbiota on $\mathrm{BC}$

Safae Terrisse and Lisa Derosa are co- first authors, and Laurence Zitvogel, Suzette Delaloge and Edoardo Pasolli are co-last authors

Edited by M. Piacentini

Supplementary information The online version contains supplementary material available at https://doi.org/10.1038/s41418021-00784-1.

Laurence Zitvogel

laurence.zitvogel@gustaveroussy.fr

Extended author information available on the last page of the article incidence or severity. Triple negative breast cancer (TNBC), whose cells do not express estrogen and progesterone hormone receptors and failed to express Her2 in in situ hybridization and immunohistochemistry, are kept in check by the immune system, as indicated by the predictive impact of tumor infiltrating lymphocytes on the efficacy of chemotherapy and prognosis [1-3] and the clinical benefit mediated by immune checkpoint inhibitors in PDL-1 expressing TNBC [4]. Accumulating evidence points to the capacity of the intestinal ecosystem, and more specifically specific health-associated bacteria commensals to dampen systemic inflammation $[5,6]$, to shape the innate and adaptive immune tonus [7-10], and reprogram the tumor microenvironment in tumor bearing mice $[11,12]$ and patients treated with immunotherapy [13, 14]. Retrospective and prospective studies conducted in lung, kidney, and melanoma cancers highlighted that antibiotics (ATB) negatively impact cancer patients' clinical outcome during therapy with anti-PD1, or anti-PDL-1 antibodies [15-18]. In parallel, metagenomics analyses scrutinizing the repertoire 
of intestinal commensalism indicated that the alpha and/or beta diversity as well as the precise taxonomic composition of the microflora dictate the prognosis of patients with melanoma [12, 14], kidney [19], and lung [11] cancer. However, no data are currently available on the impact of the gut microbiota in shaping cancer immune surveillance in the context of TNBC.

Of note, on theoretical grounds, hormone receptor positive $\left(\mathrm{HR}^{+}\right) \mathrm{BC}$ may sense the gut microbiome in a different way than TNBC. Pioneering findings implicated that long-term estrogen supplementation (which is usually applicated for alleviation of menopausal symptoms) impacted composition of gut microbiota and microbial activity, and affected estrogen metabolism in the gut of mice [20]. The diversity and composition of the gut microbiota may increase $\mathrm{BC}$ risk by modulating systemic levels of estrogens and inflammation [21].

Pioneering reports also underscored the positive effects mediated by dietary interventions on $\mathrm{BC}$ incidence or prognosis [22]. Nicotinamide that can be produced by microbial components of the gut could reduce breast tumorigenesis in a $\mathrm{T}$ and NK cell-dependent manner [23]. In mice and patients with $\mathrm{HR}^{+} \mathrm{BC}$, periodic fasting or a fasting-mimicking diet enhanced the activity of the endocrine therapeutics by lowering circulating insulin, insulin-like growth factor-1, and leptin and by inhibiting trophic signaling by the AKT-mTOR pathway [24]. Soy isoflavone influenced the composition of the gut microbiota and tumor aggressiveness in immunodeficient mice humanized by fecal transfer of BC stools and inoculated with human BC implants [25].

Thus far, very few studies investigated the taxonomic diversity of commensals in women diagnosed with early BC. Hence, time is ripe to study the microbiota repertoire, its modulation by adjuvant treatments and its relevance for the outcome and side effects of therapies. Here we unveil for two independent cohorts of early $\mathrm{BC}$ that specific metagenomics species influence $\mathrm{BC}$ prognosis and neurological, endocrine and digestive side effects of chemo-hormono-therapy, opening new avenues for the future clinical management of $\mathrm{BC}$.

\section{Results}

\section{Fecal microbiota composition at diagnosis is associated with prognosis of early $\mathrm{BC}$}

We determined the metagenomics (MG)-based composition of stools at diagnosis of early BC in a subgroup $(N=76)$ of a deep cohort called "CANTO" (for CANcer TOxicities, NCT01993498). The CANTO study aims at performing a long-term follow-up of 13250 women treated for BC over a period of ten years in order to quantify and prevent chronic toxicities related to treatment (surgery, radiation therapy, chemo-hormono-therapy) (refer to Consort diagram,
Supplementary Fig. S1). The present analysis focuses on a first set of 9595 pts Enrolled from 2012-2017 with sufficient follow-up and mature data in 2019. Among the 76 females that signed the informed consent to get MG stool analysis, the median age was 52 (Supplementary Table S1). About $43 \%$ patients presented with a T2-T3 tumor size, and $51 \%$ with a Scarff Bloom\& Richardson Grade 3 (Gr 3) adenocarcinoma (Supplementary Table S1). Up to 42 and $34 \%$ of tumors expressed HR or HER2, respectively, (24\% were TNBC) and $46 \%$ presented with lymph node involvement (at least $1 \mathrm{~N}+$ ) (Table S1). Overall, 14\% presented with AJCC stage III. The adjuvant (or neoadjuvant) therapy consisted in anthracycline-taxane (93\%), taxane- (7\%) based chemotherapy (CT) and/or hormonotherapy (62\%) (Supplementary Table S1).

The composition of the gut microbiota ecosystem was evaluated using shot gun metagenomics (MG) profiling of early BC patients pre- $(N=76)$ and post-chemotherapy (CT) $(N=45)$, the post-chemotherapy specimen being paired with the pre-CT ones. To address whether the microbial composition differed between patients presenting with a favorable (T1, Gr 1, N-, AJCC stage 1) versus unfavorable BC prognosis (T2-3, Gr 3, N+, AJCC stage II/ III), we performed univariate analysis of the variations in fecal microbial $\alpha$ diversity and provided principal coordinate analyses $(\mathrm{PCOA})$ of microbial $\beta$-diversity distances within these patient subsets. Alpha diversity (observed OTU or Shannon index) did not significantly vary among these groups (not shown). Exploratory analysis of $\beta$-diversity (measuring sample diversity) was calculated using the Bray-Curtis measure of dissimilarity. As we observed that the fecal microbiota $\beta$-diversity discriminated groups of patients according to tumor size $(<$ or $>$ pT 1 , Supplementary Fig. S2A, C), to Scarff Bloom\& Richardson grade (Gr1/2 versus Gr3, Supplementary Fig. S3A, B), to axillary node involvement $(\mathrm{N}-$ versus $\mathrm{N}+$, Supplementary Fig. S4A, C) and to TNM staging (stage I versus stage II-III, Fig. 1A, D), we utilized a supervised analysis (Partial Least Squares Discriminant Analysis (PLS-DA) to explore the variance between the groups at baseline and post-CT (Fig. 1, Supplementary Figs. S2, S3, S4). To determine the relative contribution of the relative abundance of each bacterial species (spp) to the observed group separation at baseline and post-CT, bacterial spp were ordered according to their variable importance plot (VIP) score which relies on the supervised analysis of PLS-DA, comparing groups of patients according to each $\mathrm{BC}$ prognosis factor $(<$ or $>\mathrm{pT} 1$ in Supplementary Fig. S2B, D, Gr1/2 versus Gr3 in Supplementary Fig. S3C, N- versus $\mathrm{N}+$ in Supplementary Fig. S4B, D, and stage I versus stage II/III in Fig. 1B, E).

Several consortia of commensals were found iteratively overrepresented in patient subsets bearing more aggressive tumors (associated with larger tumors at diagnosis, axillary 
A

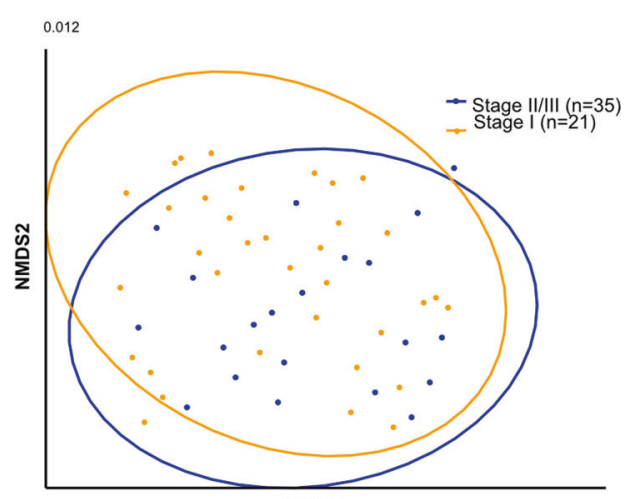

NMDS1

C

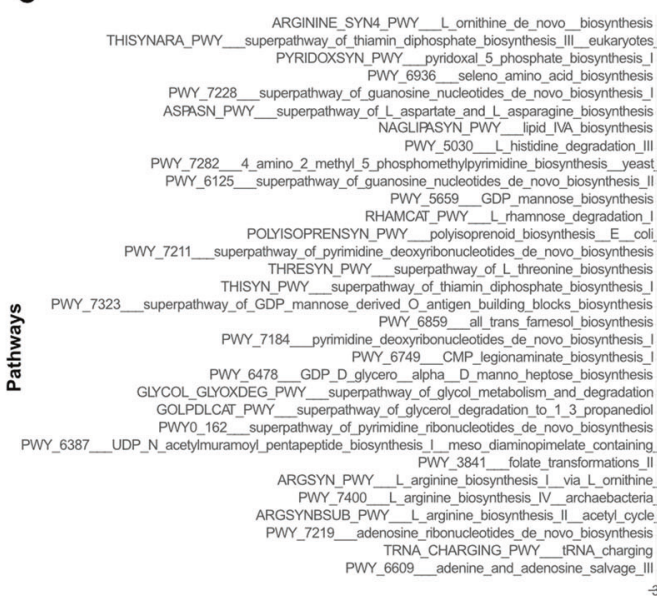

B

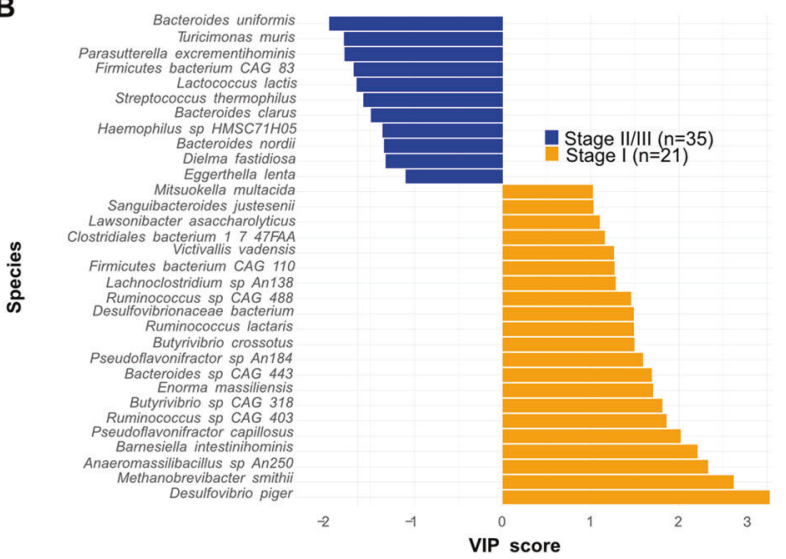

D

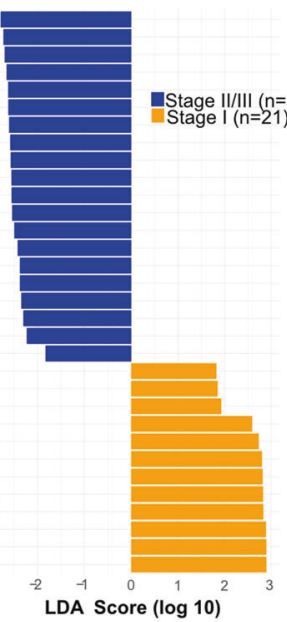

0.046

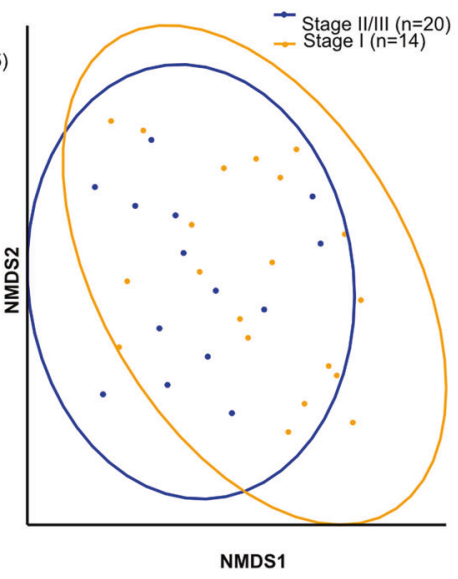

E

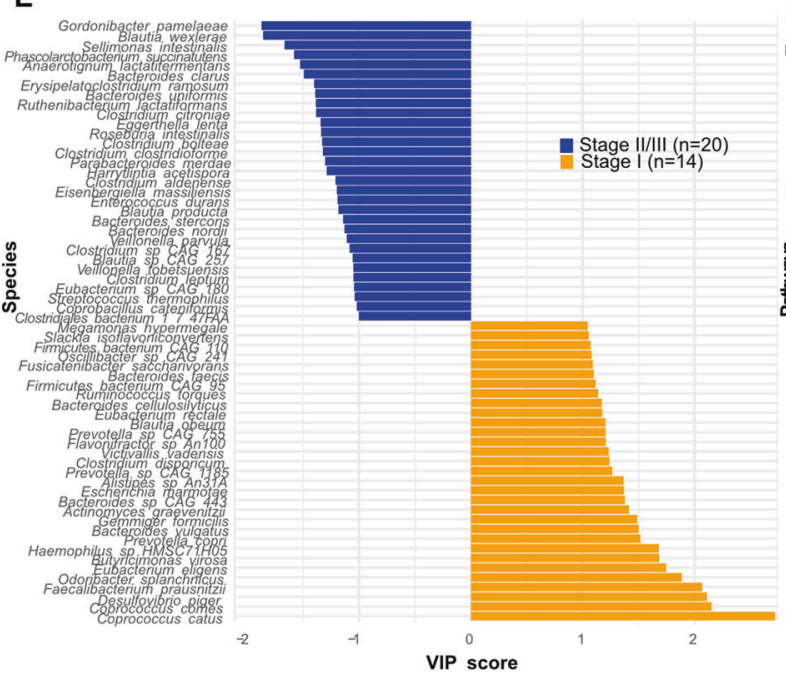

$\mathbf{F}$

PYRIDOXSYN_PWY_pyidoxal_5 phosphate_biosynthesis_

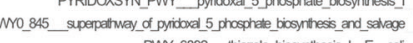
PWY_6892_tthiazole_biosynthesis_I_E_Col_ARGININE_SYN4_PWY_L__omithine_de__novo_bicsynthesis

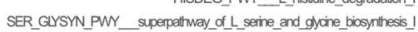

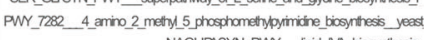

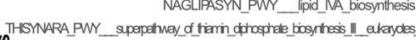
PENTOSEP_PWY pentose phosphate.pattway

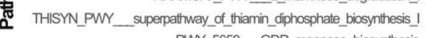
PWY_5659_GDP_mannose_bisynthesis PWY 6608 g guanosine nudeotides degradation_ll THRESYN PUY superpattway of $L$ threocine biosynthesis PWY 3001 supernathway of L isoleucine biosyntress . PUY_1861_formaldehyde_assimiation_II_RuMP_Cyde P164_PWY purne_nudeobases_degradation___anaercbic

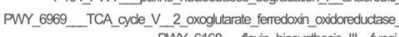
ARGSYNBSUB_PWY_L_arginine_biosynthesi__l__acety_yde_ GLUTORNPWY_L_omithine_biosynthesis ARGSYN_PWY _ L__arginine_biosynthesis__ via_L_omittine OANTIGEN_PUY_O_antigen_buiding_blocks_biosynthesis_E_ coli_ PWY_7400____arginine_biosynthesis_N__archaebacteria_

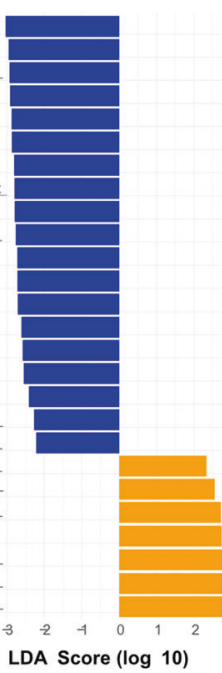

Fig. 1 Metagenomics-based stool composition is associated with TNM staging in early breast cancer (BC) patients. A and D. Betadiversity ordination plot based on principal coordinate analysis of normalized and standardized data of fecal microbiota composition before (A; pre-CT, A, $n=56)$ and after (D; post-CT, D, $n=34$ ) adjuvant chemotherapy in patients with stage I (orange) and stage II-III (blue). The p-value is indicated at the top of the $\mathrm{Y}$ axis. $\mathbf{B}$ and $\mathbf{E}$. Variable importance plot (VIP) scores were obtained within partial least square discriminant analysis by segregating stage I (orange) from stage II-III (blue), describing the most discriminant species in descending order of importance before (B; pre-CT-A, $n=56$ ) and after E; post-CT, $\mathrm{D}, n=34$ ) adjuvant chemotherapy (only bacteria with prevalence $>2.5 \%$ were taking into account). $\mathbf{C}$ and F. Differential abundances in terms of metabolic pathways between study groups (stage I vs stage II-III, pre- CT $(\mathbf{C})$ vs post-CT $(\mathbf{F})$ ) by computing the effect size from linear discriminant analysis (LDA). 
node involvement $(\mathrm{N}+)$, and UICC staging $\geq \mathrm{II})$ in fecal specimen harvested pre- and/or post-CT, such as species belonging to the Streptococcus genera ( $S$. mitis, S. vestibularis), Lachnospiraceae family members (Sellimonas intestinalis, Eisenbergiella massiliensis, Blautia wexlerae), Veillonella genus (V. atypica, V. parvula), Bacteroides spp (P. merdae, B. uniformis, B.xylanisolvens...), E. ramosum, Enterobacteriaceae family members such as Bilophila wadsworthia, Hafnia alvei and Klesiella spp, Clostridiaceae family members $(C$. spiroforme, $C$. asparagiforme, $C$. boltae) that we previously reported in advanced NSCLC and kidney cancer patients manifesting a primary resistance to immune checkpoint inhibitors [11, 19] (Fig. 1, Supplementary Figs. S2B, D and S4B, D). Conversely, healthassociated commensals, i.e., Methanobrevibacter smithii archae, Eubacteriaceae family members (such as E. eligens, E. hallii, E. rectale), A. muciniphila, Defulfovibrio piger, species belonging to the Coprococcus genus (C. comes, $C$. catus) and Collinsella genus (C. aerofaciens), B. vulgatus as well as Ruminococcaceae family members $(R$. bicirculans, $R$. lactaris) were reproducibly associated with $\mathrm{N}$ - or $\leq$ pT1 BC in longitudinal samples (Fig. 1, Supplementary Figs. S2, S3, S4B-D). Of note, A. muciniphila, C. aerofaciens, and distinct Bacteroides spp. have already been linked to favorable outcome in advanced melanoma, NSCLC and kidney cancer patients responding to immune checkpoint inhibitors [10-12, 19]. The functional pathways associated with these bacterial patterns were different between good and bad prognosis BC, but similar pre-and post-CT (Fig. 1C, F). Increased biosynthesis of L-Arginine and adenosine ribonucleotides, dominance of oxoglutarate ferredoxin oxidoreduction, and adenine-adenosine salvage were observed in favorable $\mathrm{BC}$, while biosynthesis of lipids, thiamine diphosphate, pyridoxal -5 phosphate, L-threonine and degradation of $\mathrm{L}$-histidine were associated with poorprognosis cancers (Fig. 1C, F).

Next, to further analyze the clinical relevance of these commensals associated with favorable or unfavorable prognosis, we scrutinized fecal compositional differences between these $76 \mathrm{BC}$ and 54 Italian healthy volunteers (HV), whose feces were harvested according to the same procedures and analyzed together with $282 \mathrm{HV}$-derived samples selected from referenced public metagenomes. Of note, these $282 \mathrm{HV}$ were randomly permutated several times and analyzed for data validation (as detailed in M\&M). Species with differential abundance between cancer free (HV) and BC groups were used as input for the linear discriminant analysis (LDA) to calculate an effect size (LEfSe method, LDA score $>2$ ) (Fig. 2A). We found bacterial commonalities between $\mathrm{N}$ - groups and cancer free individuals (Fig. 2B) and between $\mathrm{N}+$ and $\mathrm{BC}$ status (Fig. 2C). Hence, we identified 7 MG species associated with both a cancer -free status and with a more favorable prognosis, in case of cancer (mostly $\mathrm{N}$ - and/or TNM staging I) in post-CT specimen, such as $C$. comes and $C$. catus, Collinsella aerofaciens, E. rectale, M. smithii, or $\mathrm{MG}$ recovered in both pre- and post- $\mathrm{CT}$ feces (such as $B$. crossotus, C. aerofaciens) (Fig. 2B). Conversely, we identified 7 commensals characterizing $\mathrm{BC}$ patients and associated with worse prognosis in pre- or post-CT specimens such as $B$. uniformis and $C$. bolteae $(\mathrm{N}+$ and TNM staging $>\mathrm{I}), P$. merdae, $C$. asparagiforme, $R$. intestinalis, $B$. intestinihominis (Fig. 2C).

Next, we attempted to validate these findings in a second independent cohort of $\mathrm{BC}$ patients treated with neoadjuvant $\mathrm{CT}$ enrolled in CANTO $(n=17$ pre-CT, 10 post-CT) (Supplementary Table S1). Here again, the LEfSe method allowed to segregate baseline stool compositions of $\mathrm{BC}$ patients with post-chemotherapy $\mathrm{pN}+$ versus $\mathrm{pN}$ - status (Fig. 2D) as well as pathological complete responders (stage 0 ) from non-complete responders (stage I-II) (Supplementary Fig. S3D). B. vulgatus, Blautia obeum and Dorea formicigenerans that were found overrepresented post-CT in stage I BC (Fig. 1E, Fig. 2A) stood out among the few taxons associated with the $\mathrm{pN}$ - status (metastasis-free axillary lymph nodes post-neoadjuvant CT) (Fig. 2D). In contrast, $8 \mathrm{MG}$ species described in $\mathrm{N}+$ or stage II-III post-CT (i.e., E. ramosum, C. bolteae, C. citroniae, C. aldenense, C. clostridioforme, Blautia wexlerae, Sellimonas intestinalis, Eisenberghiella massiliensis) and overabundant in $\mathrm{BC}$ patients as compared to HV (Fig. 2A) were also significantly associated with the $\mathrm{pN}+$ status in postneoadjuvant CT specimen, but not with cancer immunohistochemical subtype (Fig. 2D, Supplementary Fig. S3D).

Altogether, using four complementary methodologies of comparisons, sampling pre-CT, sampling post-CT, cancer -free versus cancer bearing status, and adjuvant versus neoadjuvant settings, we conclude that the intestinal ecosystem represents another variable to consider in women at early $\mathrm{BC}$ diagnosis, that possibly influences prognosis (as based on correlations with tumor size, histological grade, axillary LN involvement and staging). Of note, in this cohort of 76 early BC, MG stool composition was associated with the presence of axillary metastases in univariate analyses identically to the classical clinical prognostic parameters (Supplementary Table S2).

\section{Adjuvant chemotherapy modified the $\beta$ diversity of fecal composition in early BC}

To characterize changes (if any) in microbial composition over the course of 8 cycles of CT, we first analyzed the variations in fecal microbial $\alpha$ diversity (Fig. 3A) and performed PCoA of microbial $\beta$ diversity distances (Fig. 3B). Compared with baseline (prior to $\mathrm{CT}$ ), $\alpha$ diversity (richness index) increased after CT (Fig. 3A, $p=0.033$ ). When 
A.

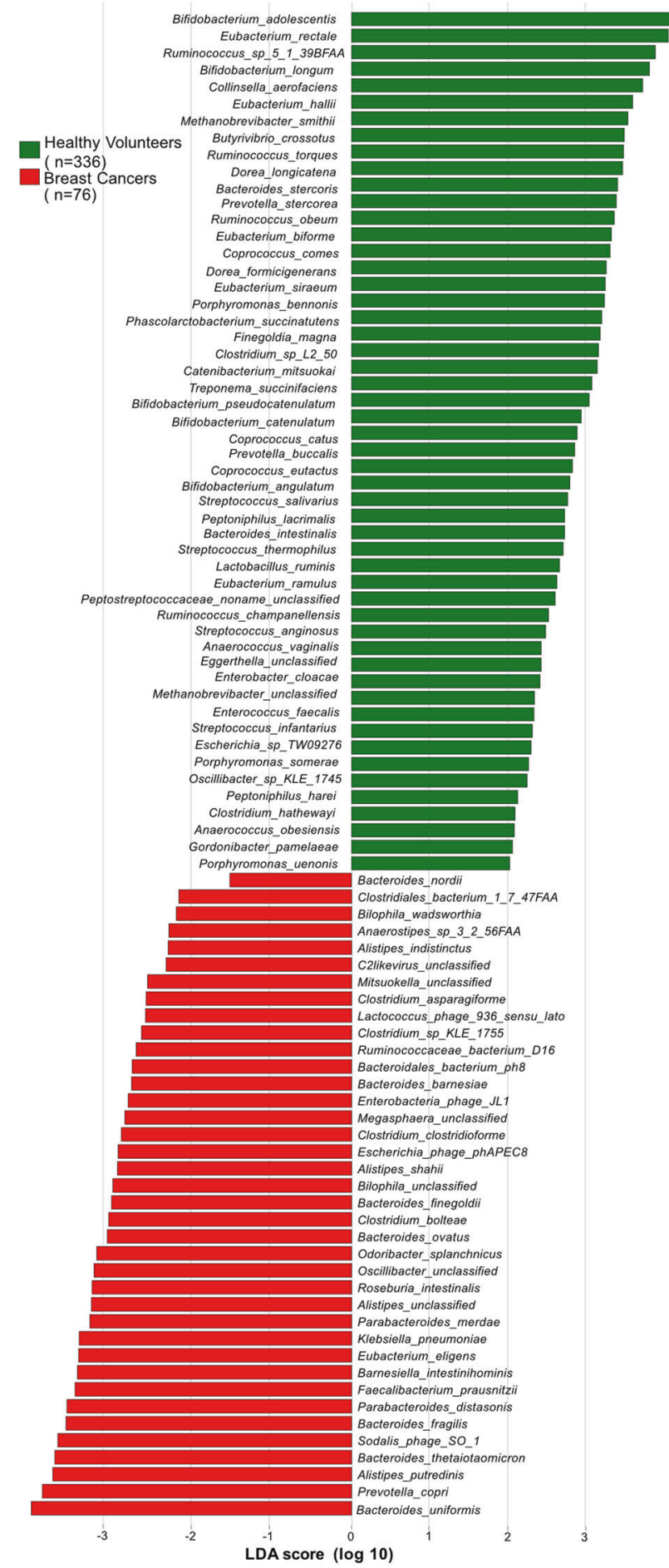

B.

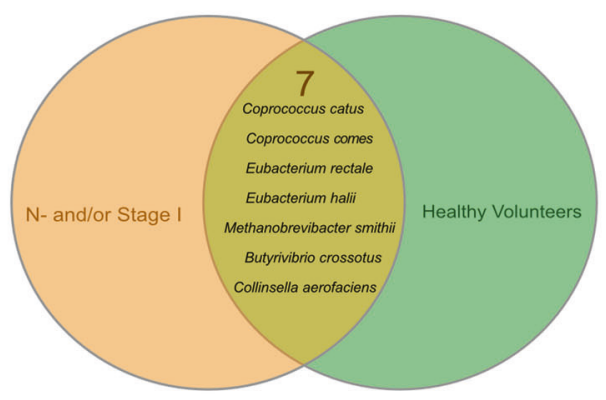

C.

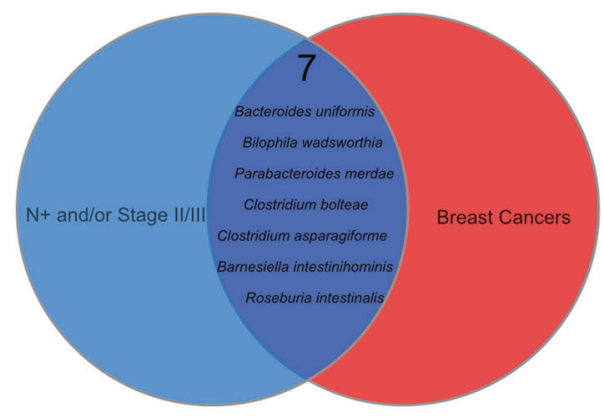

D.

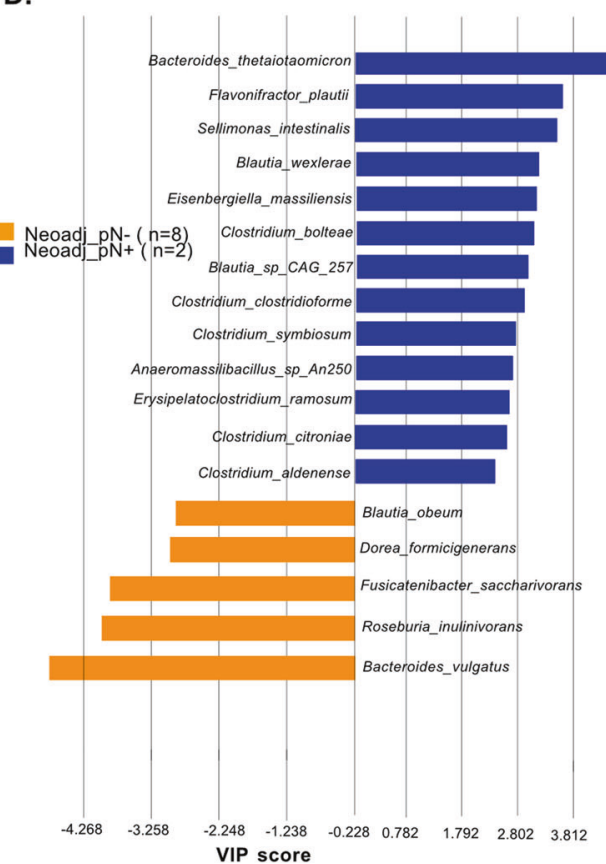

Fig. 2 Metagenomics analyses of stool samples from $\mathrm{BC}$ patients compared with healthy volunteers (HV). A LEfSe (Linear discriminant analysis of effect size) method was used to detect differential abundant species (only bacteria with a prevalence $>2.5 \%$ were considered) between (green bars, $n=336$ ) and BC patients (BC, red bars, $n=76$ ). Venn diagram describing the listing and the numbers of bacterial species in common between no pathological lymph node involvement $(\mathrm{pN}-)$ and $\mathrm{HV}(n=7, \mathbf{B})$ and in common between pathological lymph node involvement $(\mathrm{N}+)$ and $\mathrm{BC}(n=7, \mathbf{C}) . \mathbf{D}$. VIP scores by comparing species abundance according to the pathological lymph node involvement (no pathological lymph node involvement, pN-, orange; pathological lymph node involvement, $\mathrm{pN}+$, blue) in stools collected after neoadjuvant CT $(n=10)$. 
A.

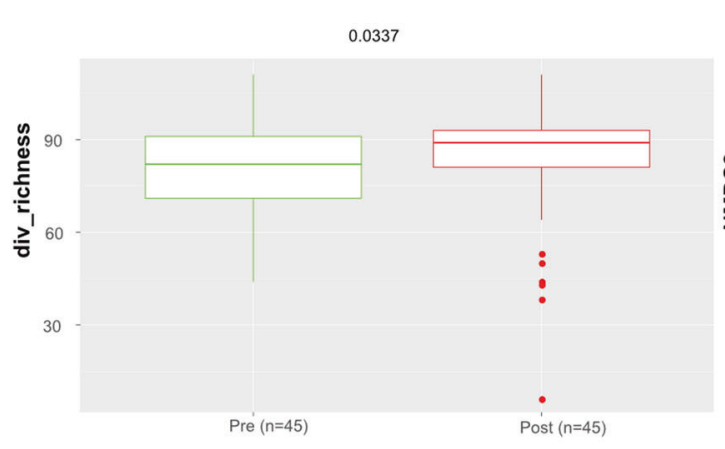

B.

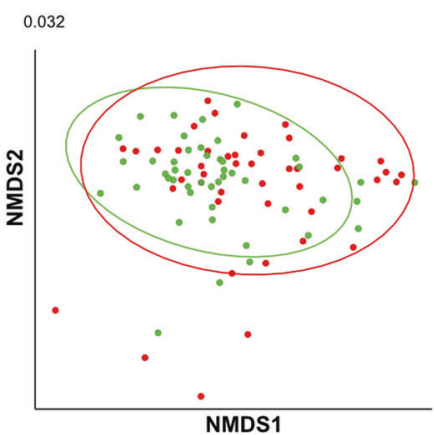

c.

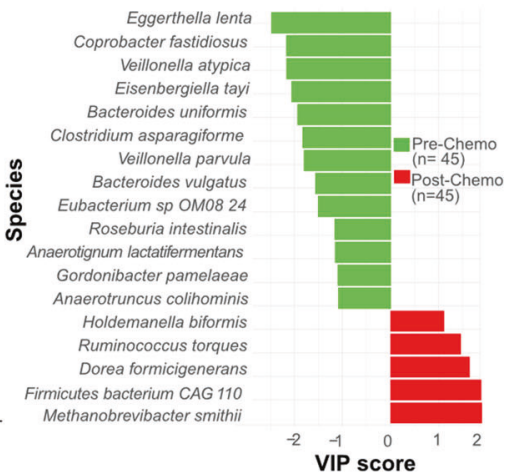

D.

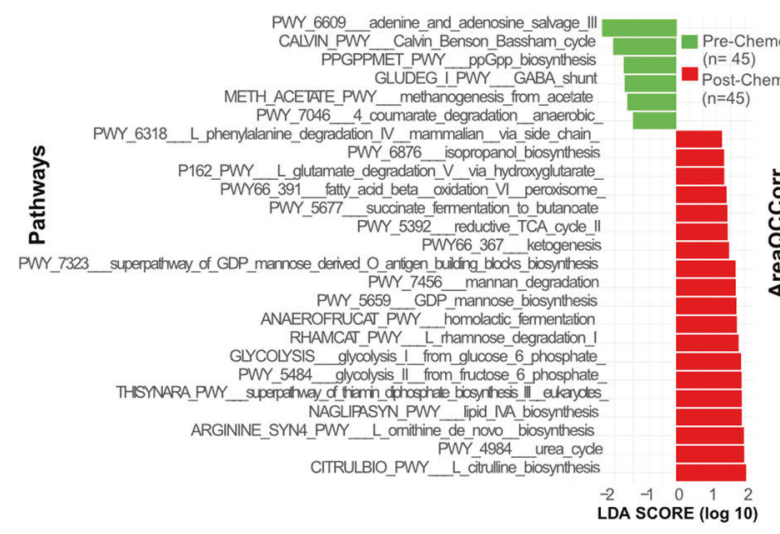

E.

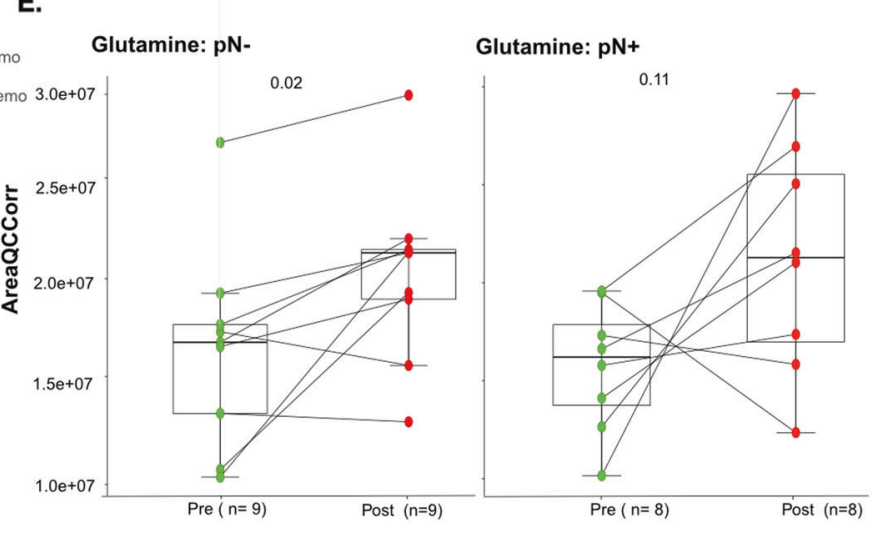

Glutamine: $\mathrm{pN}+$

G.

F.

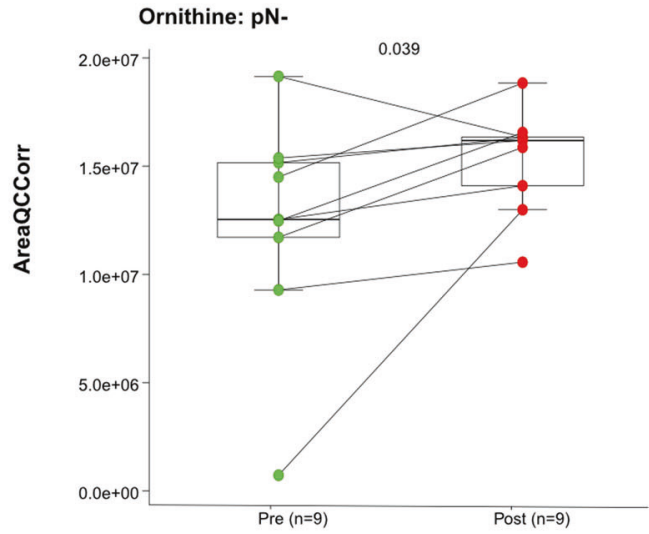

Ornithine: $\mathrm{pN}+$

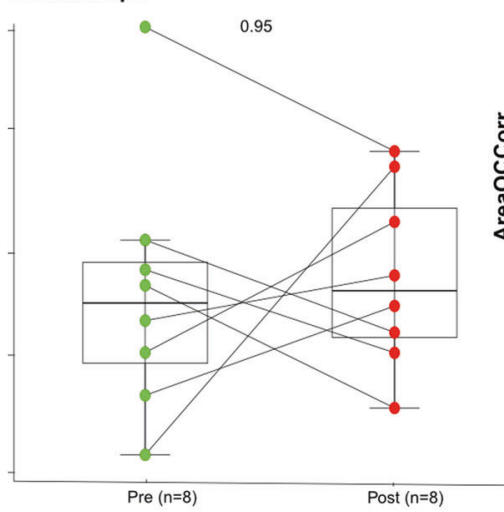

Kyn/Trp: pN-

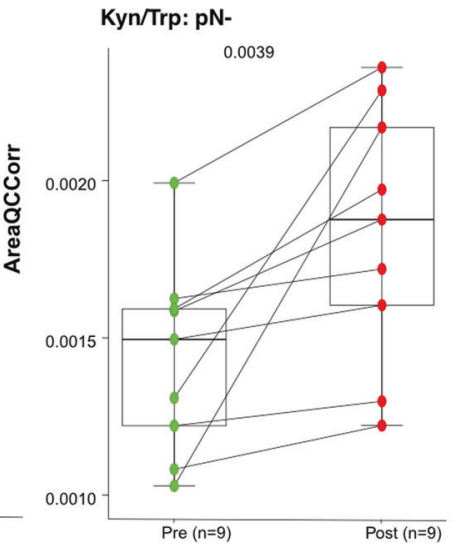

Fig. 3 Chemotherapy significantly affected fecal composition in a paired sub-group analysis. A. Alpha diversity in terms of richness in longitudinal and paired stool samples $(n=45)$ collected before (pre$\mathrm{CT}$, green) and after (post-CT, red) CT. B. Beta-diversity ordination plot based on principal coordinate analysis of normalized and standardized data of fecal microbiota composition in pre-CT (green) and post-CT (red) stools. Most discriminative species (C; through VIP (implemented within partial least square discriminant analysis) and pathways (D; through LEfSe) by differentiating between pre-CT and post-CT samples stools. lasma levels of metabolites through high

considering $\beta$ diversity variations in a paired manner, we identified significant shifts in the microbial composition over time (Fig. 3B, $p=0.032$ ). Chemotherapy increased the dimensional metabolomics according to pathological lymph node involvement (no pathological lymph node involvement, pN-; pathological lymph node involvement, $\mathrm{pN}+$ ) in terms of Glutamine (in $\mathrm{pN}$ patients, $n=18$, left panels; and $\mathrm{pN}+$ patients, $n=16$, right panels). $\mathbf{E}$ Ornithine (in pN-patients, $n=18$, left panels; and $\mathrm{pN}+$ patients, $n=$ 16, right panels) $\mathbf{F}$ and Kynurenine to Tryptophan (Kyn/Trp) ratio in $\mathrm{pN}$ - patients monitored in patients pre-CT, (green) and post-CT, (red), $n=18$ (G). $P$ values are indicated at the top of the $Y$ axis (Wilcoxon test).

relative abundance of health-related MG species (such as Methanobrevibacter smithii archae associated with $\mathrm{HV}$ and pN- status in BC (Fig. 2B), D. formicigenerans (overabundant 
in $\mathrm{HV}$, Fig. 2A), R. torques (associated with stage I BC, Fig. 1B, E pre-et post-CT, Fig. 3C). In contrast, CT tended to reduce the over-representation of bacteria associated with $\mathrm{BC}$ diagnosis and unfavorable prognosis (such as $C$. asparagiforme, B. uniformis, Eggerthella lenta, species from the Veillonella genus) (Fig. 3C). Of note, chemotherapy did not significantly affect the relative abundance and prevalence of the immunogenic species A. muciniphila, associated with $\leq \mathrm{pT} 1$, in pre-CT stool samples (not shown). Interestingly, the functional pathways associated with bacterial gene patterns were influenced by $\mathrm{CT}$, which increased L-ornithine biosynthesis, glycolytic intermediates (glucose-6-phosphate and fructose-6-phosphate), L-glutamate degradation, lipid biosynthesis, and ketogenesis (Fig. 3D).

To validate these metabolic patterns associated with fecal content, we performed a metabolomic analysis of the plasma in 29 BC females ( 26 pre- and 29 post-CT samples) compared with age matched HV. Indeed, we confirmed that glutamine and L-ornithine plasma levels increased during CT, mostly in pN- (Fig. 3E, F). Conversely, the tryptophane/kynurenine degradation pathway, known to be involved in lymphocyte exhaustion and associated with advanced staging in BC stools (Supplementary Fig. S5A) was stimulated by CT, mostly in pN- (Fig. 3G).

$\mathrm{L}$-methionine (increased in stool gene patterns within patients >T1) tended to increase in the plasma of stage II-III patients post-CT (Supplementary Fig. S5B, C, right and left panel respectively). Krebs cycle metabolites were higher in stools of $\mathrm{pN}+$ versus $\mathrm{pN}$ - and increased in plasma post- $\mathrm{CT}$ in $\mathrm{pN}+$ (Fig. S5B). The iNOS regulator dimethylarginine was increased in the plasma post $\mathrm{CT}$ at later stages of $\mathrm{BC}$, while L-arginine biosynthesis was found in stools of good prognosis BC (Supplementary Fig. S5D).

Altogether, significant changes occurred in the stool composition after 8 cycles of adjuvant anthracyclines and taxanes, expanding commensals associated with more favorable prognosis, the metabolic pathway of polyamines (L-Arg, L ornithine) and glutamine synthesis.

\section{Gut microbiota and chemotherapy-related side effects in CANTO}

The LEfSe method was used to compare the abundance of all bacterial clades pre- and post-CT according to the occurrence of toxicity at 12 months, considering neurological, gastrointestinal, rheumatological, or metabolic (BMI) disorders (Supplementary Table S3). The $\alpha$-diversity of the gut microflora was not significantly different between patients with or without side effects pre-CT, but varied postCT according to BMI, diarrhea and constipation (Supplementary Figs. S6A, C and S7B, D). In univariate analysis, $\beta$-diversity post-CT significantly predicted neurological side effects (comprising paresthesia, peripheral sensory neuropathy, memory disorders, concentration defects) (Fig. 4A, $p=0.013$, Supplementary Table S3), as well as overt weight gain (Supplementary Fig. S6), constipation, diarrhea or hot flashes, while rheumatological side effects were not significantly associated with the gut composition (Supplementary Fig. S7). 51 patients had neurological side effects at month 12 while 16 did not. Bacterial taxa with differential abundance between study groups complaining or not from neurological side effects were used as input for the LDA. The bacteria that were associated with neurological symptoms belonged to the Clostridiaceae family (i.e C. symbosium, C. bolteae, C. spiriforme, C. aldenense, $C$. citroniae, C. asparagiforme and E. ramosum) (Fig. 4B). They were also retained in the LEfSe model in prechemotherapy samples, as an internal validation (not shown). Most of these taxonomic species that were associated with neurological symptoms post-CT were clinically relevant for the dismal prognosis (as outlined Fig. 1 and Fig. 2A-D and Supplementary Figs. S2, S3, i.e, C. bolteae, C. spiroforme, E. ramosum, E.coli, B. uniformis, B. thetaiotaomicron, Blautia wexlerae, Eggerthella lenta, Ruthenibacterium lactatiformans).

Conversely, some of the bacteria underrepresented in patients who were less susceptible to develop neurological side effects also featured in the list of commensals associated with good prognosis and/or healthy status (C.aerofaciens, C.comes, D.piger, $R$. torques, B. crossotus). However, it is noteworthy that most of the bacteria associated with dismal prognosis that were dominant pre-CT (and disadvantaged by $\mathrm{CT}$ ) were especially abundant in the stools of patients who did not suffer from weight gain $(C$. bolteae, C. clostridioforme, C. aldenense, C. hathewayi, $E$. ramosum, E. coli, B. uniformis, Eisenbergiella tayi, spp from the Veillonella genus and Streptococci spp.) (Supplementary Fig. S6, Supplementary Table S4).

The microbiome functional potential was analyzed at the genetic level in the MG data base of our CANTO cohort using the HUMAnN3 database [26]. Neurological side effects were associated with intestinal functional pathways involving mainly energy production with an enrichment in the glycolysis pathways, L-histidine degradation, fatty acid biosynthesis and beta-oxidation, as described for stools from patients with dismal prognosis. In contrast, microbial genes coding for enzymes involved in ribonucleotide de novo biosynthesis, polyamine biosynthesis and the GABA shunt were linked to neuroprotection (Fig. 4C).

\section{Relevance of the findings in gut humanized avatar mouse models of $B C$}

In order to show a cause effect relationship between the gut composition and tumor aggressiveness, we took advantage of avatar mouse models, which are first conditioned by 
A

0.013

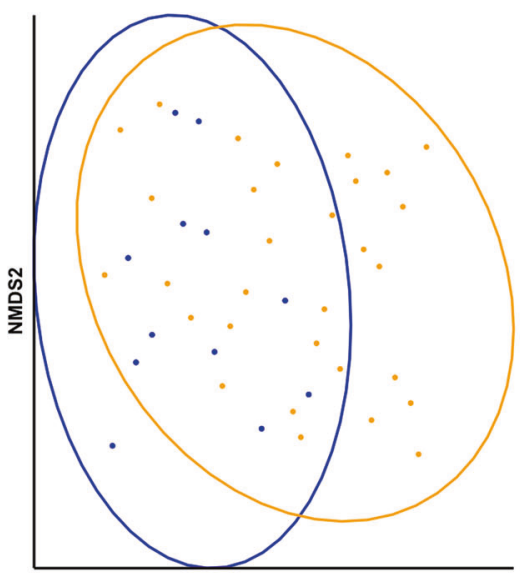

NMDS1
B
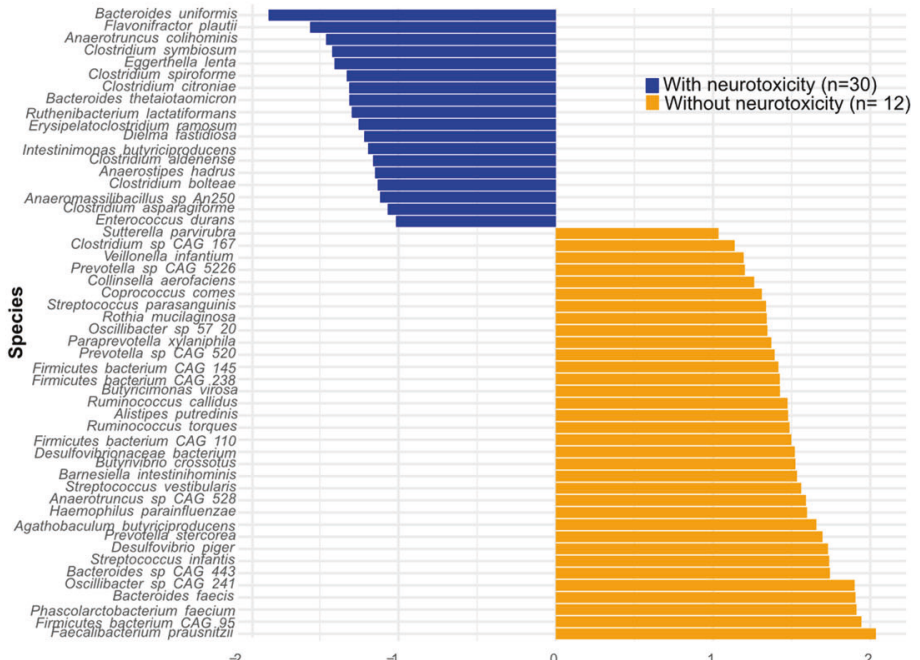

Without neurotoxicity $(n=12)$

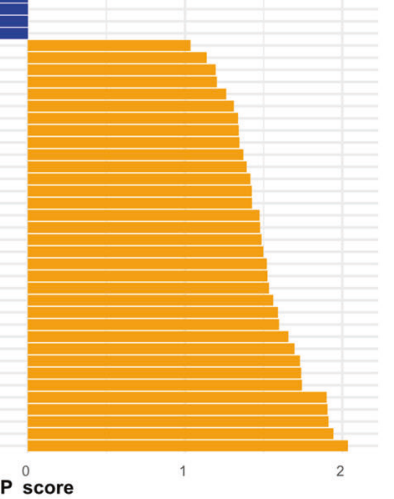

C

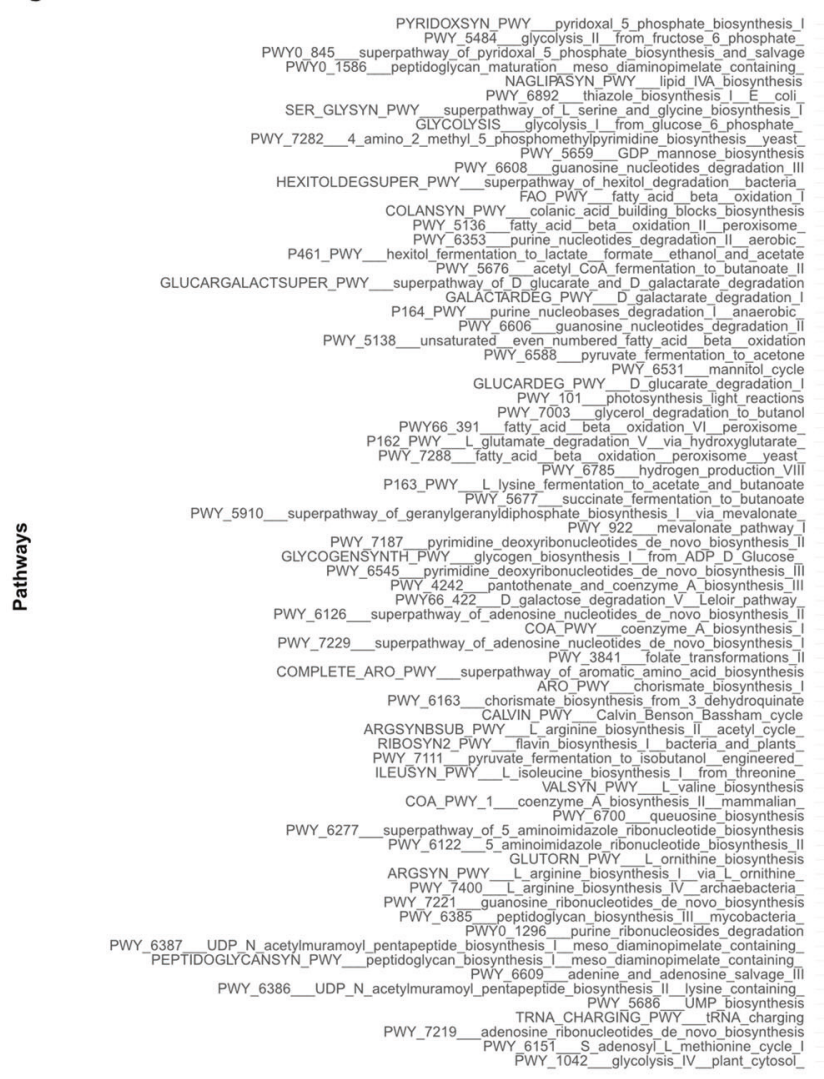

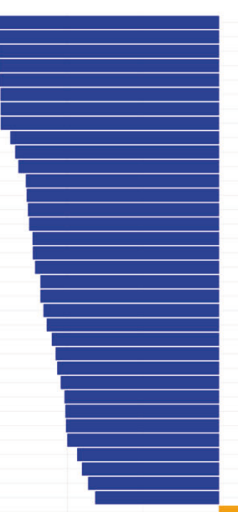

VIP score

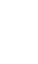

. 
A

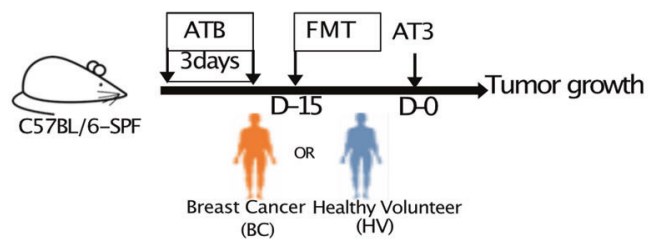

B

\begin{tabular}{|l|l|}
\hline Status & Characteristics \\
\hline BC1 & TN, Stage II, Tils: $5 \%$ \\
\hline BC2 & HR+, Stage II, Tils: $10 \%$ \\
\hline BC3 & TN, Stage II, Tils: $25 \%$ \\
\hline BC4 & TN, Stage III, Tils: $50 \%$ \\
\hline BC5 & TN, Stage II, Tils: $5 \%$ \\
\hline HV1 & Fr, $27 y$, man \\
\hline HV2 & Ita, $29 y$, man \\
\hline HV3 & Ita, 55y, woman \\
\hline HV4 & Ita, $41 y$, man \\
\hline HV5 & Ita, $40 y$, man \\
\hline HV6 & Ita, $36 y$, woman \\
\hline HV7 & Ita, $43 y$, woman \\
\hline
\end{tabular}

\section{C}

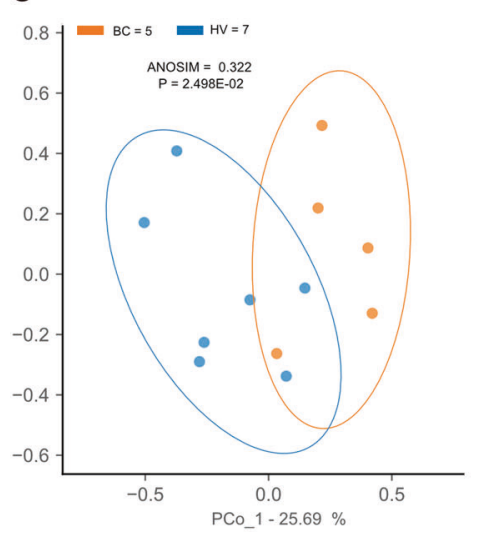

E
D

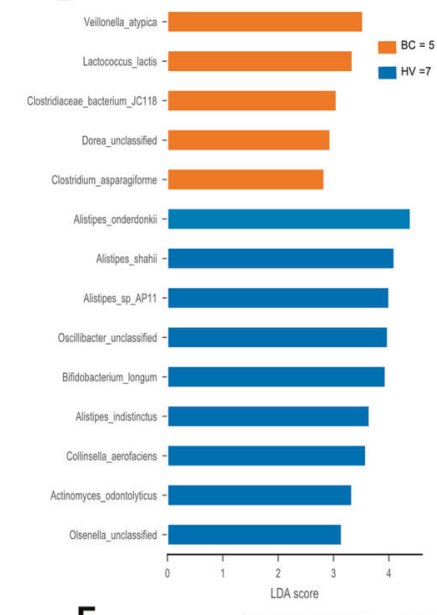

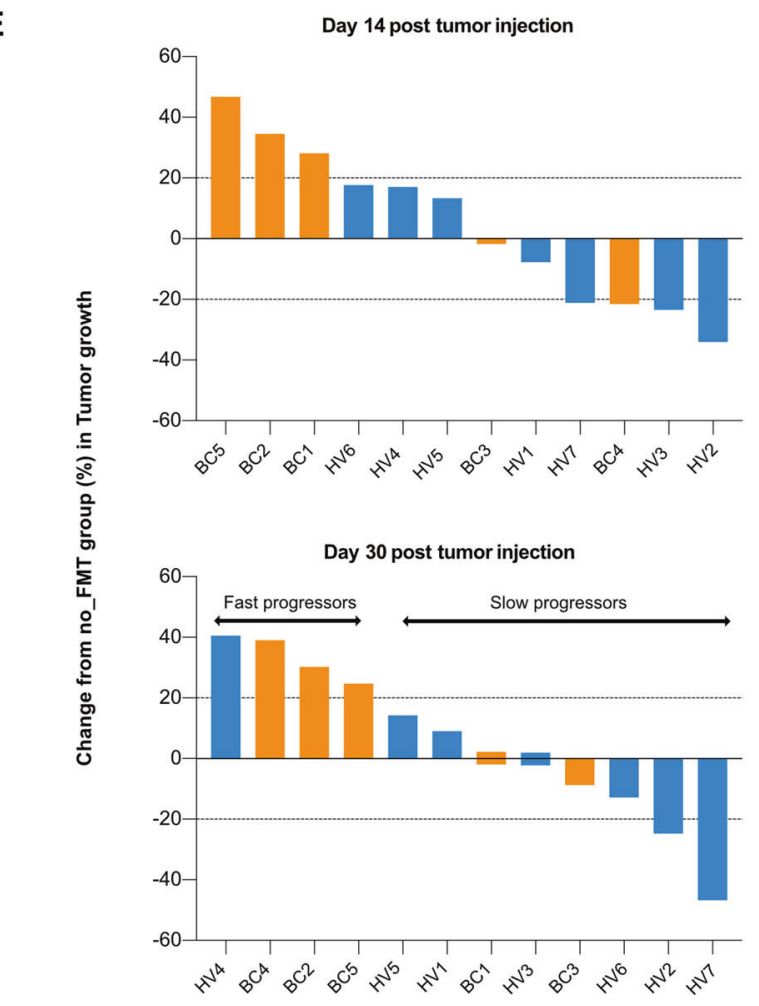

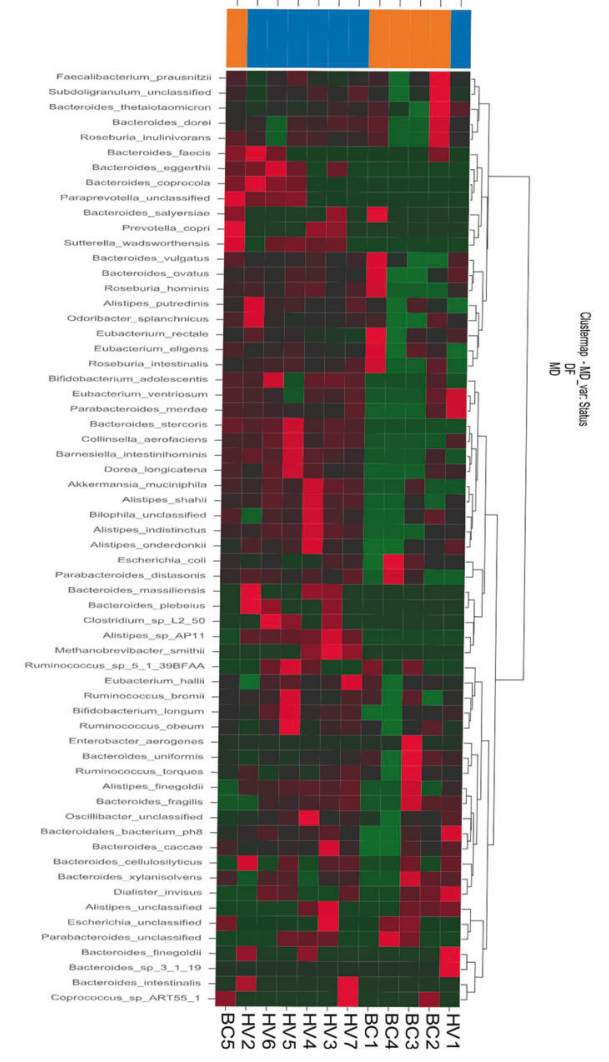

broad-spectrum antibiotics to eradicate the endogenous mouse microbiota and then "humanized" by fecal microbial transplantation (FMT). Such "humanized" immunocompetent C57BL/6 mice were inoculated with syngeneic transplantable AT3 BC cells as previously described [27] to investigate tumor development in the absence or presence of chemotherapy (Fig. 5A). We performed oral gavage with
FMT using stools from seven independent $\mathrm{HV}$ and five $\mathrm{BC}$ patients at diagnosis (Fig. 5B) for whom the MG analyses were available (Fig. 5C, D). Fifteen days later, we challenged recipient avatar mice with a lethal dose of AT3 to follow BC growth kinetics in comparison with non FMT "eubiotic" mice reared in specific pathogen-free conditions. The LEfSe method was used to compare the abundance of 
Fig. 5 Gut humanized avatar AT3 tumor bearing mice mirrored patient prognosis. A Experimental setting of avatar mice. Fecal microbial transplantation (FMT) of feces from Healthy Volunteers (HV) or early breast cancer patients (BC) into AT3 tumor bearing C57BL/6 mice. Arrows details treatments. Each experiment contained 6 mice/groups for each FMT and each donor et was performed at least two times. B Details on clinical and pathological characteristics of human donors for FMT (five early BC and seven HV). C Betadiversity ordination plot based on principal coordinate analysis of normalized and standardized data of donors' fecal microbiota composition in $\mathrm{BC}$ (orange) and $\mathrm{HV}$ (blue) to analyze the most discriminant species in between the two donor groups. D LEfSe (Linear discriminant analysis of effect size) differentiating $\mathrm{BC}$ and $\mathrm{HV}$, describing the 14 most discriminant species in descending order of importance in 5 early BC pre-CT versus $7 \mathrm{HV}$ stools used for FMT in AT3 tumor bearing mice. E AT3 tumor size in each group of FMT at 2 time points, 14 days (Top panel) and 30 days (low panel) after tumor inoculation. Means are depicted for 5-6 mice/group. The tumor size changes $(\%)$ were compared with special pathogen free conditions (No FMT) at the same timepoint. A deviation of $+20 \%$ compared with special pathogen free conditions (No FMT) was defined as "fast versus slow" progressors. Each experiment has been performed once for each donor with 12 FMT performed in a blinded manner. F Heatmap of the non-supervised hierarchical clustering of microbiota composition of stool samples across all 12 donors of FMT. Colors (from green to dark red) shows the row scaled relative abundance of each taxon across all samples.

all bacterial clades according to the clinical status (HV versus BC). Bacterial taxa with differential abundance between the two study groups were used as input for the linear discriminant analysis (LDA) to calculate an effect size (LEfSe analysis) that revealed a relative dominance of Alistipes spp. (A. shahii being associated with stage I BC (Supplementary Fig. S3) and B. longum and C. aerofaciens in PCoA, in HV compared with BC (Fig. 5C, D), in accordance with the contrast observed between $\mathrm{HV}$ and $\mathrm{BC}$ described in Fig. 2 and S3. When we monitored AT3 tumor progression, we observed two groups of stools that mediated opposite effects, one group that tended to mitigate natural tumor progression, called "slow progressors" (dominated by HV2, HV3, HV7) while the other group called "fast progressors" that tended to accelerate the natural kinetics (encompassing BC2, BC4, BC5, HV4-5) (Fig. 5E). The non-supervised hierarchical clustering of fecal MGS of the FMT donors could also segregate slow from fast progressing tumor bearers (Fig. 5F). A closer examination of the taxonomic species overrepresented in slow (versus fast) progressors revealed several members of the Eubacterium genus (E. rectale, E. eligens), A. muciniphila, Actinobacteria class (B. longum, C. aerofaciens) and Alispites shahii, which were already identified in patient stools from patient with stage I or N- BC pre-or post-CT. In contrast, fast progressors received fecal material containing $\mathrm{MG}$ species associated with dismal prognosis (such as B. uniformis, B. xylanivolvens, B. intestinalis, Fig. 5E).
Next, driven by previous reports showing that ATB negatively affect the efficacy of CT based on oxaliplatin [28] or cyclophosphamide (CTX) [7, 8], we addressed the question as to whether the microbiota composition could modulate the efficacy of CTX-based therapy in AT3 BC bearing "humanized" mice (Fig. 6A). "Humanization" by FMT from four BC patients and five HV (listed in Fig. 5B), demonstrated that CTX-mediated anticancer effects were affected by the intestinal microbiota. In the vast majority of mice bearing a human BC-associated gut microflora, the tumoricidal activity of CTX was barely significant, contrasting with mice that obtained an FMT from $\mathrm{HV}$ (Fig. 6B-D). Taking advantage of the coprophagic behavior of rodent, we treated mice "humanized" with BC feces and that were doomed to fail CTX-based chemotherapy by cohousing with "humanized mice" benefiting from a HVderived microbiota or, alternatively, by oral gavage with $\mathrm{HV}$-derived feces. In 3 independent experiments, HVderived microbiota allowed to restore the CTX-mediated anticancer effects (Fig. 6B-D, Fig. 5B).

Altogether, these experiments demonstrate that the composition of the fecal microbiota influences the natural growth of BC (without therapy), as well as the response of $\mathrm{BC}$ to CTX-based CT. Thus, variations in intestinal microflora is not a mere correlate of $\mathrm{BC}$ progression but rather influence the course of the disease in a causative fashion.

\section{Discussion}

To our knowledge, this study offers the first description of the gut microbiota repertoire defined by shotgun $\mathrm{MG}$ sequencing of fecal samples in early $\mathrm{BC}$ at diagnosis and its dynamics after adjuvant chemotherapy. The data presented herein suggest that (i) distinct commensals from the gut microbiome impact $\mathrm{BC}$ prognosis in patients and $\mathrm{BC}$ tumor aggressiveness in mice, (ii) chemotherapy can tilt the balance between favorable or unfavorable species, and iii) selected commensals may affect the probability of developing neurological side effects.

In contrast to previous reports focusing in metastatic melanoma or advanced NSCLC receiving PD-1-targeting immune checkpoint blockade (ICB), $\alpha$ diversity was not predictive of favorable $\mathrm{BC}$ prognosis, nor of side effects $[12,14,29]$. In contrast, $\beta$ diversity of the gut microbiota was associated with tumor grading (but not breast cancer subtype), LN involvement and staging, as well as with neurological side effects at 12 months. At diagnosis (and regardless of chemotherapy), fecal composition containing a relative dominance of the trio of $C$. bolteae, $C$. asparagiforme, and B. uniformis was associated with axillary lymph node invasion, as previously described for resistance 
A
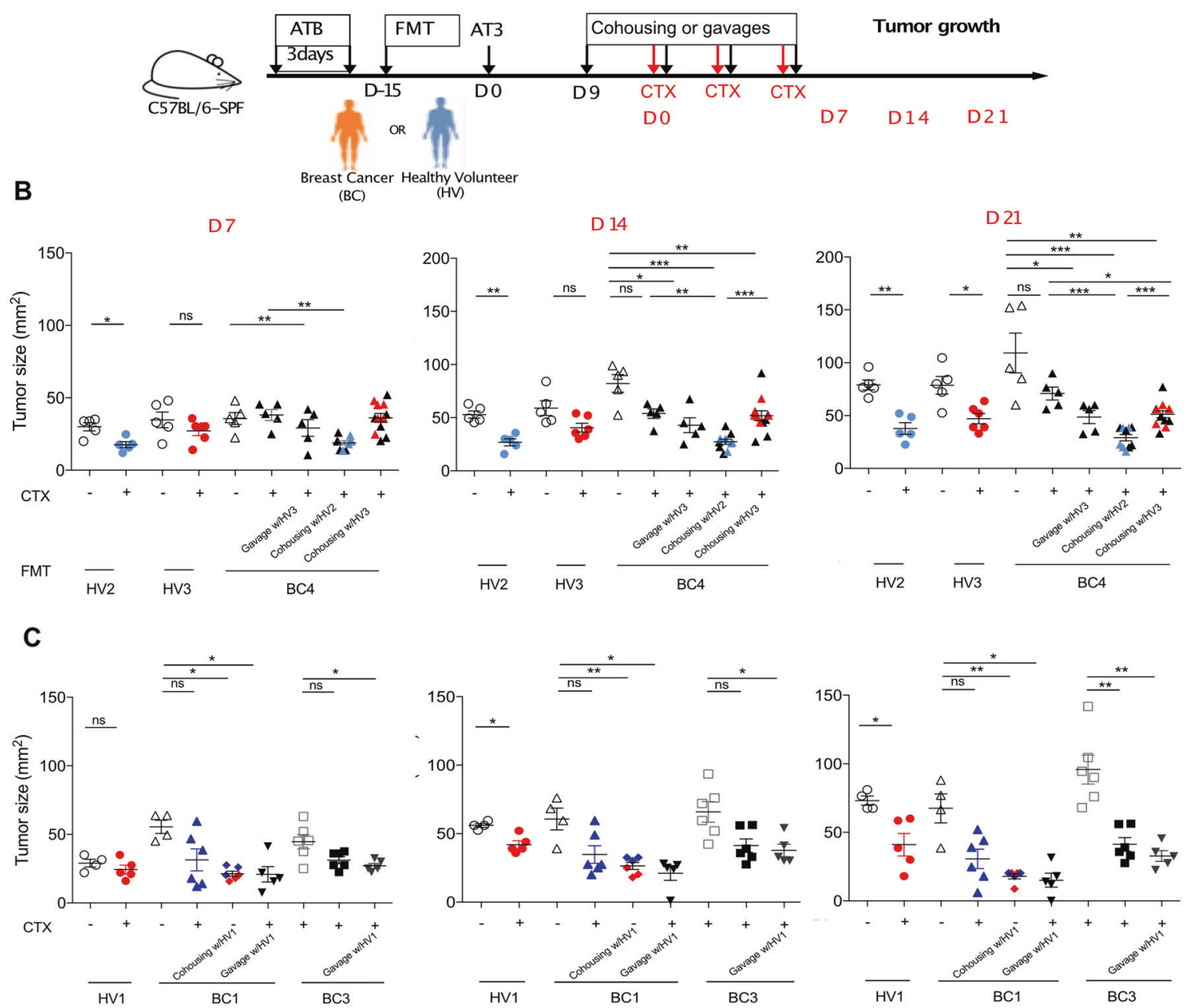

D
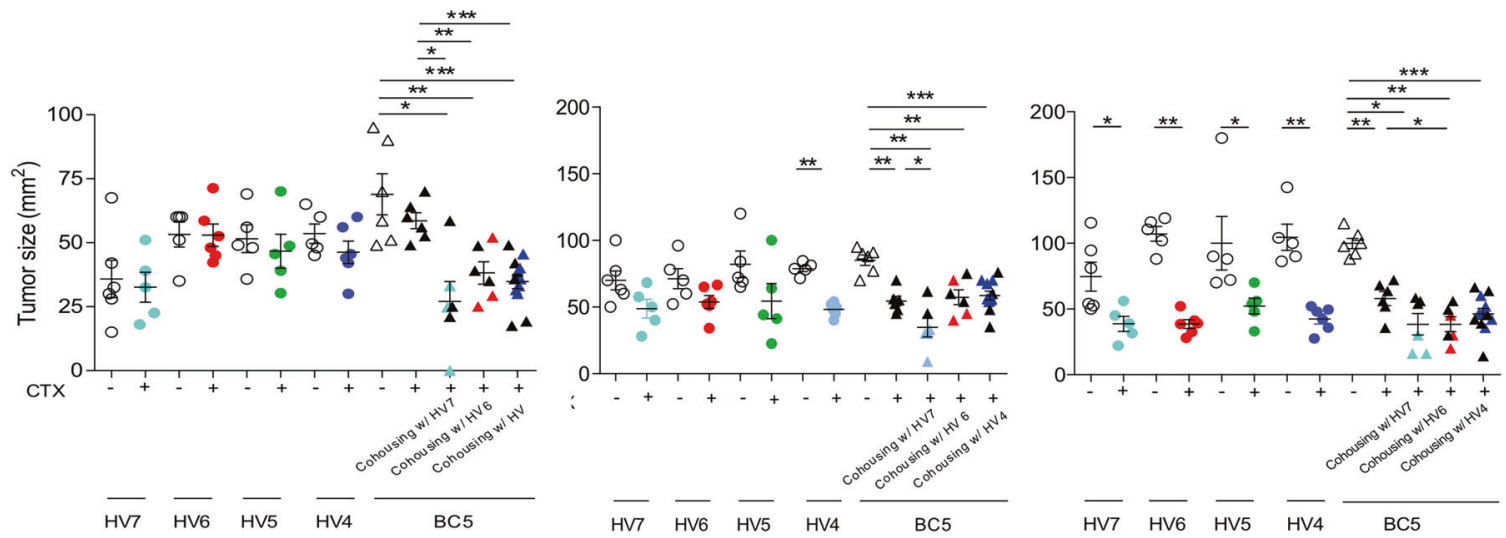

Fig. 6 CTX-mediated tumoricidal activity is affected by the gut microbiota composition in avatar AT3 bearers. A. Experimental Setting. As in Fig. 5, FMT using stools from HV or early BC was performed following 3 days of ATB in specific pathogen-free (SPF) C57BL/6 mice. Two weeks later, AT3 breast tumors were subcutaneously inoculated. 9 days after, mice were randomly assigned to cohousing or FMT with different donors (HV or BC) and concomitantly treated with 4 intra peritoneal (i.p.) injections of cyclophosphamide (CTX). Tumor size was recorded for each mouse on days D7, D14, D21 after CTX starting. 5-6 mice/groups for each FMT and each donor. B-D. Means AT3 tumor sizes \pm SEM at different timepoits (D7, D14, and D21 after CTX starting) showing the BC induced dysbiosis and compensation with FMT or cohousing with different HV (described in Fig. 5) resulting in rescued response to CTX (each color representing one donor, details on donors charactheristics are showed on Fig. 5B). Each experiment included 5-6 mice per group. ANOVA statistical analyses of means and SEM: ${ }^{*} p<$ $0.05, * * p<0.01, * * * p<0.001$, ns: non-significant. 
to ICBs in advanced NSCLC and kidney cancers [19, 27]. In contrast, E. rectale, $M$. smithii, C. comes or $C$. catus., and Actinobacteria (C. aerofaciens) that were associated with the $\mathrm{HV}$ status and dominant in feces of $\mathrm{N}$ - and/or stage I BC patients also correlated with favorable prognosis of patients with kidney cancer or melanoma treated with ICBs [12, 19]. Of note, $C$. aerofaciens was also retrieved by LEfSe analysis of the "humanized" gut from AT3 bearing mice exhibiting slower tumor progression (Fig. 5). Our data are in line with a pioneering shot gun MG analysis performed on stools from 18 premenopausal and 44 post-menopausal women diagnosed with BC [30]. This latter study indicated significant differences between $\mathrm{BC}$ and healthy controls post-menopause, with increased $\alpha$ diversity and proinflammatory Enterobacteriaceae members (E. coli, Klebsiella spp), and a concomitant decrease in Eubacterium eligens in BC patients (correlating with decreased tumorinfiltrating lymphocytes, TILs) compared with HVs.

Moreover, $\mathrm{HR}+\mathrm{BC}$, which constitute the largest group in our study, are not expected to be under strong $\mathrm{T}$ cellbased immunosurveillance. HR $+\mathrm{BC}$ are poorly infiltrated with TILs. Of note, our work did not reveal any significant correlation between the gut microbiota and the density of TILs (not shown). One might speculate that the intestinal ecosystem of women doomed to develop this BC subtype might be relatively poor in commensals associated with a strong immune tonus, such as A. muciniphila, B. fragilis, $E$. hirae, Bifidobacteria spp. Eubacteriaceae family members. Supporting this notion, A. muciniphila was found associated with small tumor size (pT1) BC in our study. More than $55 \%$ women with $\mathrm{BC}$ lacked detectable A. muciniphila, in line with the association of $\mathrm{BC}$ with type 2 diabetes and high BMI, two conditions that are also associated with the absence of A. muciniphila [31].

Importantly, we found that chemotherapy drastically shifted the microbiome composition, tilting the balance towards favorable commensals in a subset of patients. Hence, in two BC subcohorts, we observed that several bacterial spp. associated with ATB uptake or resistance to ICB (i.e Clostridia such as C. hathewayi, C. clostridioforme, C. symbosium, C. aldenense, C. citroniae, E. ramosum, as well as Veillonella spp, and Eisenbergiella massiliensis or E. tayi) which were associated with $\mathrm{N}+$ status at diagnosis and neurological side effects, became less abundant after adjuvant or neoadjuvant treatment with anthracylines and taxanes. In contrast, CT favored the colonization or residence of favorable commensals such as Methanobrevibacter smithii, D. formicigenerans, Blautia obeum, or R. torques. Importantly, the gut humanized avatar AT3 bearers that were subjected to FMT containing species from the Eubacterium genus (E. rectale, E. eligens, E. ventriosum) or $C$. aerofaciens exhibited a reduced tumor growth rate compared with eubiotic control mice. A pioneering study [32] focusing on the possible involvement of microbiota changes in the beneficial effects of metronomic (versus standard dosing of) capecitabine in 15 metastatic HER2 negative BC described differences in commensalism between the two CT regimens and the association of Slackia and Blautia obeum with poor and favorable clinical outcome, respectively. Therefore, it will be important to validate the capacity of specific chemotherapeutic agents to eliminate unfavorable bacteria or to expand favorable commensals in future clinical studies.

Very few reports previously evocated a potential impact of the gut microbiome on $\mathrm{BC}$ risk or prognosis [33]. One study investigated stools from 31 early BC patients by realtime qPCR of genes encoding for distinct bacterial families or species-related 16S rRNA [34]. The authors of this study concluded that Clostridium leptum and $C$. coccoides clusters, which express $\beta$-glucuronidases favoring the reabsorption of free estrogen, were enriched in patients with stage II-III (as opposed to stage I) BC [34]. Our study supports the previously described deleterious role of Clostridiacae family members $(C$. citroniae, $C$. bolteae, $C$. clostridioforme, C. symbosium, C. aldenese, C. hathewayi, C.asparagiforme) across various malignancies (kidney, lung, and breast cancers) and therapies (ICBs, anthracyclines, taxanes, CTX).

Mechanistically, an earlier report pioneered the idea that microbiota can dictate the prognosis of extraintestinal ovarian and breast cancers via an effect on Toll-like receptor 5 (TLR5) [6]. TLR5 recognition of flagellinexpressing commensals may favor systemic tumor -promoting inflammation and malignant progression of BC. Thus, TLR5 signaling drives recirculation of IL-6, mobilizes of myeloid suppressor cells and elicits immunosuppressive galectin-1-producing $\gamma \delta \mathrm{T}$ cells [6].

More importantly, overt commensal dysbiosis might cause systemic inflammation that induced collagen deposition and accelerated fibrosis of the normal and tumor mammary gland through infiltration of myeloid cells [35]. Besides promoting systemic inflammation, gut dysbiosis may alter whole body metabolism. Zhu et al. explored functional features of the gut microbiota in pre- and postmenopausal control in $\mathrm{BC}$ females, and reported that amino acid transport and nucleotide synthesis dominated the KEGG modules in pre-menopause, while LPS and betaoxidation were mostly found post-menopause [30]. Of note, our study pointed to the overrepresentation of lipid betaoxidation modules in BC patients prone to develop neurological side effects. Conversely, our study suggested an association between protection from taxane-induced neurological side effects and the overrepresentation of purinergic pathways in the gut microbiota. These latter finding might be interpreted to suggest that increased production of the neuroprotective purine adenosine (which reportedly 
favors neurotransmission [36], affects neuro-immunity [37], maintains the blood-brain barrier [38] and avoids neurodegeneration [39-41] through effects on A1, A2A, A2B, and A3 adenosine receptors [42]) might prevent the taxaneinduced neuropathy by direct effects on neural circuitries. Moreover, the metabolic pathway of polyamines is associated with better prognosis and neuroprotection in our cohort. Polyamines constitute major products of the colonic microbiota, as shown after oral administration of the immunogenic commensal A. muciniphila [43]. Polyamines can improve the intestinal barrier function [44] and cancer immune-surveillance [45].

Finally, CT stimulate intestinal ketogenesis. Since ketone bodies favor immunosurveillance [46], this effect may contribute to the anticancer effects of CT.

Altogether, the findings reported in this study may have important consequences for the future clinical management of early BC patients. First, monitoring the presence and abundance of favorable versus unfavorable commensals may be important to predict the efficacy of adjuvant or neoadjuvant chemotherapy. Second, compensatory therapies aimed at providing immunogenic commensals may turn cold $\mathrm{HR}^{+} \mathrm{BC}$ into TIL-enriched tumor microenvironment amenable to ICBs and/or more sensitive to chemotherapies. Such an effect has been achieved with live biotherapeutics in preclinical models [9, 27, 47]. Supporting the notion that metabolism can be kept in check by commensals, a preparation of oral pre-and probiotics (containing E. halii, A. muciniphila, and inulin) reduced postprandial glucose levels in type 2 diabetes patients in a randomized double blind placebo controlled trial [48], confirming earlier trials [49]. Thus, it is possible to treat human disease by intervening on the gut microbiota. Third, diet interventions may represent yet another way to influence the gut microbiota. While promising approaches such as fasting or ketogenic [46] diets have direct cell autonomous or indirect immune effects on cancer, it remains to be determined whether fasting-induced microbial shifts may participate in the immune control of $\mathrm{HR}^{+} \mathrm{BC}$ [24]. Thus, future studies must determine the best strategy to manipulate the intestinal microbiota to improve the clinical prognosis and quality of life of BC patients.

\section{Materials and methods}

\section{Clinical study}

We enrolled patients from CANTO trial (for CANcer TOxicities NCT01993498), a prospective clinical cohort including non-metastatic breast cancer (BC). The CANTO study aims at performing a long-term follow-up of 13250 women treated for BC over a period of ten years in order to quantify and prevent chronic toxicities related to treatment (surgery, radiation therapy, chemo-hormono-therapy). The present analysis focuses on a first set of 9595 pts enrolled from 2012 to 2017 with sufficient follow-up and mature data in 2019, of which 76 were included in the biological microbiota sub-study (refer to Consort diagram, Supplementary Fig. S1). Patients stools were collected before and after chemotherapy (adjuvant or neoadjuvant) at home, in kits including an anaerobic generator (Biomerieux). Samples were frozen $4-24 \mathrm{~h}$ later at $-80^{\circ} \mathrm{C}$ at Gustave Roussy Cancer Campus in plastic tubes (Plastic vessel by 1000 Sarstedt) with or without RNA later. Plasma samples were collected for 29 BC females: 26 samples before chemotherapy and 29 after chemotherapy. In parallel, clinical data were collected prospectively at three time points: at diagnosis (baseline); 3-6 months after the end of primary treatment (i.e., primary surgery, chemotherapy, or radiotherapy, whichever came last), and then 1 year post primary treatment. Antihuman epidermal growth factor receptor (HER2) therapy and hormonal therapy could be ongoing, if indicated. Toxicity data were collected at 3-6 months and 1 year after the end of primary treatment. Clinical characteristics and toxicities are described in supplemental tables (Supplementary Tables 1, 2, and 3). CANTO is coordinated by UNICANCER, the National Cooperative Group of French Cancer Centers. The study was approved by the national regulatory authorities and ethics committee (IDRCB: 2011-A01095-36, 11-039). All patients enrolled in the study provided written informed consent, including consent for the biological data collection.

Furthermore, we used the feces of 54 Italian HV from Instituto Nazionale dei Tumori center, whose feces were harvested according to the same procedures and analyzed together with 282 HV-derived samples selected from referenced public metagenomes.

\section{Metagenomics analysis}

Total fecal DNA was extracted as described $[50,51]$ and sequenced using ion-proton technology (ThermoFisher) resulting in $22.7 \pm 0.9$ million (mean $\pm \mathrm{SD}$ ) single-end short reads of 150-base-long single-end reads as a mean. Reads were cleaned using [52]. Alien Trimmer in order (i) to remove resilient sequencing adapters and (ii) to trim low quality nucleotides at the $3^{\prime}$ side using a quality and length cut-off of 20 and $45 \mathrm{bp}$, respectively. Cleaned reads were subsequently filtered from human and other possible food contaminant DNA (using Human genome RCh37-p10, Bos Taurus and Arabidopsis thaliana and an identity score threshold of 97\%). MetaPhlAn3 pipelines were used to profile fastq files deriving from shotgun sequencing in order to describe the bacterial species present in each sample [53]. These pipelines are based on a gene-reference database, 
thus protein-coding gene markers deriving from the collection of the $+100 \mathrm{k}$ bacterial genomes (which we especially focused on) deposited in NCBI or ENA archives. With few gene markers available for each bacterial species (ranging from 50 to 400 ) it was thus possible to ascertain their presence and relative abundance (expressed in the interval $0-100 \%$ ) within assembled shotgun samples. For the MetaOMineR analyses the gene abundance profiling was performed using the 9.9 million gene integrated reference catalog of the human microbiome [54]. Filtered highquality reads were mapped with an identity threshold of $95 \%$ to the 9.9 million-gene catalog using [55]. Bowtie 2 included in METEOR software [56]. The gene abundance profiling table was generated by means of a two-step procedure using METEOR. The gene abundance table was processed for rarefaction and normalization and further analysis using the MetaOMineR package [57]. The gene abundance table was rarefied to 13 million reads per sample (a threshold chosen to include all samples, but 1 with 12.5 million reads) by random sampling of 13 million mapped reads without replacement. The resulting rarefied gene abundance table was normalized according to the FPKM strategy (normalization by the gene size and the number of total mapped reads reported in frequency) to give the gene abundance profile table. Metagenomic species (MGS) are co-abundant gene groups with more than 500 genes corresponding to microbial species. 1436 MGS were clustered from 1267 human gut microbiome samples used to construct the 9.9 million-gene catalog [54], as described [58]. Differentially abundant MGS between different patients' groups were selected using the Wilcoxon test $(p<0.05)$. Microbial gene richness (gene count) was calculated by counting the number of genes that were detected at least once in a given sample, using the average number of genes counted in ten independent rarefaction experiments. MGS richness (MGS count) was calculated directly from the MGS abundance matrix. For the MetaPhlAn3 analyses fastq files were cleaned/filtered as described above and underwent an additional filtering for possible human contaminants (reference database GRCh37/hg19) and contextual quality control using KneadData. This wrapper entangles Bowtie2 ("-very-sensitive" and "-dovetail" settings) to rule out contaminant sequences and Trimmomatic (sliding window 20, min-length 50) to exclude low-quality reads. Filtered reads underwent MetaPhlAn3 pipeline (default settings) for unambiguous taxonomic classification and to generate a table of relative abundances for bacterial, archael, eukaryotic species. Only taxa that were present in at least $5 \%$ of all samples were kept for downstream analysis. Measurements of $\alpha$ diversity (within sample diversity) in terms of Shannon index, Simpson index, and richness were calculated at species level using the diversity function in the $\mathrm{R}$ environment. Exploratory analysis of $\beta$-diversity (between sample diversity) was calculated using the BrayCurtis measure of dissimilarity and represented in PCoA.

Supervised PLS-DA and the subsequent VIP were used to find out the most discriminant bacterial species among the cohorts, having a prevalence cutoff $>=5 \%$. Two-tailed Mann-Whitney $U$ and Kruskall Wallis tests were employed using the function in the $\mathrm{R}$ environment to assess significance for pairwise or multiple comparisons, respectively, considering a $P$ value $\leq 0.05$. Longitudinal analysis differences were assessed by Mann-Whitney $U$ test. All $P$ values underwent a Benjamini-Hochberg two-stages false detection rate at $10 \%$. Gene-family and pathway-relative abundances were generated by HUMAnN3 [53]. Univariate analyses were performed using LEfSe [59] to estimate the effect size of features that were statistically significant among groups. The biomarkers with the highest effect sizes were reported and discussed. Cross-correlation Pearson matrices for network analysis (metric $=$ Bray-Curtis, method $=$ complete linkage) were generated and visualized with Gephi version 0.9.2.

\section{Metabolomics analysis}

\section{Plasma sample preparation}

Plasma samples $(50 \mu \mathrm{L})$ were mixed with $500 \mu \mathrm{L}$ of the of ice-cold extraction mixture, allowing protein precipitation and metabolites extraction, then vortexed and centrifuged $\left(10 \mathrm{~min}\right.$ at $\left.15000 \mathrm{~g}, 4^{\circ} \mathrm{C}\right)$. Supernatants were collected, split in 4 fractions, and treated according to the protocols described in Viltard et al. [60]. Briefly, upper phase of supernatant was split in three parts: $150 \mu \mathrm{L}$ were used for GC-MS experiment in injection vial, $40 \mu \mathrm{L}$ were used for the SCFA (Short Chain Fatty Acids) HPLC-MS method, and $120 \mu \mathrm{L}$ were used for others UHPLC-MS experimentations. The 4th fraction together with the sample pellet were re-extracted with an equal volume of $2 \%$ SSA (in $\mathrm{meOH})$, vortexed and centrifuged $\left(10 \mathrm{~min}\right.$ at $\left.15000 \mathrm{~g}, 4^{\circ} \mathrm{C}\right)$ for polyamines detection.

\section{Widely-targeted analysis of intracellular metabolites}

GC/MS. GC-MS/MS method was performed on a 7890B gas chromatography (Agilent Technologies, Waldbronn, Germany) coupled to a triple quadrupole 7000C (Agilent Technologies, Waldbronn, Germany) equipped with a High sensitivity electronic impact source operating in positive mode [60].

\section{Targeted analysis of bile acids}

Targeted analysis was performed on a RRLC 1260 system (Agilent Technologies, Waldbronn, Germany) coupled to a 
QTRAP 6500+ (Sciex) equipped with an electrospray source operating in negative mode. Gas temperature was set to $4500^{\circ} \mathrm{C}$, with ion source gas 1 and 2 set to 30 and 70 , respectively [60].

\section{Targeted analysis of polyamines}

Targeted analysis was performed on a RRLC 1260 system (Agilent Technologies, Waldbronn, Germany) coupled to a Triple Quadrupole 6410 (Agilent Technologies) equipped with an electrospray source operating in positive mode. The gas temperature was set to $350^{\circ} \mathrm{C}$ with a gas flow of $12 \mathrm{l} /$ $\min$. The capillary voltage was set to $3.5 \mathrm{kV}$ [60].

\section{Targeted analysis of short chain fatty acid}

Targeted analysis was performed on a RRLC 1260 system (Agilent Technologies, Waldbronn, Germany) coupled to a Triple Quadrupole 6410 (Agilent Technologies) equipped with an electrospray source operating in negative mode. Gas temperature was set to $350{ }^{\circ} \mathrm{C}$ with a gas flow of $12 \mathrm{~L} / \mathrm{min}$. Capillary voltage was set to $4.0 \mathrm{kV}$ [60].

\section{Pseudo-targeted analysis of intracellular metabolites}

The profiling experiment was performed with a Dionex Ultimate 3000 UHPLC system (Thermo Scientific) coupled to a Q-Exactive (Thermo Scientific) equipped with an electrospray source operating in both positive and negative mode and full scan mode from 100 to $1200 \mathrm{~m} / \mathrm{z}$. The QExactive parameters were: sheath gas flow rate 55 a.u., auxiliary gas flow rate 15 a.u., spray voltage $3.3 \mathrm{kV}$, capillary temperature $300^{\circ} \mathrm{C}, \mathrm{S}$-Lens RF level $55 \mathrm{~V}$. The mass spectrometer was calibrated with sodium acetate solution dedicated to low mass calibration [60].

\section{Statistical analysis}

All targeted and pseudo-targeted treated data were merged and cleaned with a dedicated R (version 3.4) package (@Github/ Kroemerlab/GRMeta). Calculations and statistical tests were performed using R v3.4. Wilcoxon-Mann-Whitney test was used to assess differences in concentration between two different groups. Data representation was performed with softwares R v3.6 and Rstudio v1.2.1335 using tidyverse, dplyr, ggplot2, ggpubr, complexheatmap, and corrplot packages.

\section{Experimental tumor model and treatments}

- Mice: All animal experiments were carried out in compliance with French and European laws and regulations. The local institutional animal ethics board and French Ministère de la Recherche approved all mouse experiments (permission numbers: 2016-0494646, 2018-078-17530). Experiments were performed in accordance with Government and institutional guidelines and regulations. Female C57BL/6 were purchased from Harlan (France). Mice were used between 7 and 12 weeks of age. All mouse experiments were performed at the animal facility in Gustave Roussy Cancer Campus where animals were housed in specific pathogen-free conditions.

- Tumor models and CTX treatment: C57BL/6-derived AT-3 mouse breast tumor cell line was processed as previously described [61]. To examine s.c. tumor growth, mice were inoculated subcutaneously on the right flank with $5 \times 10^{5}$ of cells and tumor size monitored. Once tumors were established (20-40 $\mathrm{mm}^{2}$ ). Mice were injected weekly i.p with $100 \mathrm{mg} / \mathrm{kg}$ of cyclophosphamide (CTX, Endoxan, Baxter provided by Gustave Roussy) for three injections or equivalent volume of PBS. Mice were randomized based on their tumor sizes to homogenize the groups.

Mice were excluded of the analysis if the reached the endpoint established by ethical committee such as tumor size or tumor necrosis.

- Antibiotics: Mice were treated with an antibiotic cocktail (ATB) containing ampicillin $(1 \mathrm{mg} / \mathrm{ml})$, streptomycin (5 $\mathrm{mg} / \mathrm{ml})$, and colistin $(1 \mathrm{mg} / \mathrm{ml})$ (Sigma-Aldrich), in the drinking water of mice. Antibiotic activity was confirmed by cultivating fecal pellets resuspended in BHI $+15 \%$ glycerol at $0.1 \mathrm{~g} / \mathrm{ml}$ on COS (Columbia Agar with $5 \%$ Sheep Blood) plates for $48 \mathrm{~h}$ at $37^{\circ} \mathrm{C}$ in aerobic and anaerobic conditions. ATB were given during 3 days and FMT was performed the next day.

- FMT: FMT was performed by thawing fecal material. Two hundred $\mu \mathrm{L}$ of the suspension was then transferred by oral gavage into ATB pretreated recipient. In addition, another $100 \mu \mathrm{L}$ was applied on the fur of each animal. Two weeks after FMT, tumor cells were injected subcutaneously. Mice were treated with CTX or PBS and randomly assigned to $+/-$ oral gavage of fecal samples from $\mathrm{HV}$ into humanized mice with $\mathrm{BC}$ patients FMT. The experimenter was blinded for the healthy volunteer or breast cancer status.

- Cohousing experiments: Two weeks after FMT, tumor cells were injected subcutaneously. Mice were treated with CTX or PBS, then randomly assigned to +/cohousing with humanized mice with $\mathrm{HV}$ or BC.

The experimenter was blinded to the healthy or breast cancer status of the patients. Each experiment included 5-6 mice per group. Data are representative of at least two independent experiments. 


\section{Statistical analysis}

Data analyses and representations were performed either with the statistical environment, Microsoft Excel or Prism 6 (GraphPad).

The Mann-Whitney $U$ test was used to compare two independent groups, whereas nonparametric Kruskal-Wallis tests were implemented for multiple groups. For comparisons between patient groups, the Mann-Whitney $U$ test $P$ value was used.

All tests were two-sided and $P$ values $<0.05$ were considered to be statistically significant.

Data from in vivo experiments were expressed as the mean \pm standard error of the mean (SEM). Data are representative of at least two independent experiments.

\section{Data availability}

Raw data for the metagenomes newly sequenced in this study are available in NCBI-SRA under the BioProject PRJNA718520.

Acknowledgements LZ and GK are supported by the Ligue contre le Cancer (équipe labellisée); Agence Nationale de la Recherche (ANR) -Projets blancs; ANR under the frame of E-Rare-2, the ERA-Net for Research on Rare Diseases; AMMICa US23/CNRS UMS3655; Association pour la recherche sur le cancer (ARC); Association "Le Cancer du Sein, Parlons-en!"; Cancéropôle Ile-de-France; Chancelerie des Universités de Paris (Legs Poix), Fondation pour la Recherche Médicale (FRM); a donation by Elior; European Research Area Network on Cardiovascular Diseases (ERA-CVD, MINOTAUR); Gustave Roussy Odyssea, the European Union Horizon 2020 Project Oncobiome; Fondation Carrefour; High-end Foreign Expert Program in China (GDW20171100085), Institut National du Cancer (INCa); Inserm (HTE); Institut Universitaire de France; LeDucq Foundation; the LabEx Immuno-Oncology (ANR-18-IDEX-0001); the RHU Torino Lumière; the Seerave Foundation; the SIRIC Stratified Oncology Cell DNA Repair and Tumor Immune Elimination (SOCRATE); and the SIRIC Cancer Research and Personalized Medicine (CARPEM). The CANTO study is supported by the French Government under the "Investment for the Future" program managed by the National Research Agency (ANR), grant $n^{\circ}$ ANR-10-COHO-0004. ST is financially supported by Institut national de la santé et de la recherche médicale (INSERM)_-Plan Cancer 2014-2019.

Author contributions S.D. took part of the project from the sample preparation to data acquisition and analytic report. He's in charge of the storage of the raw data.

\section{Compliance with ethical standards}

Conflict of interest The authors LZ, GK declare being founders of everImmune. FP declares grants and other from Abbvie, grants and other from Amgen, grants and other from Celgene, grants and other from Novartis, grants and other from Roche, other from Seattle Genetics, other from PriME/Medscape, personal fees from Chugai, grants and other from Daiichi-Sankyo, other from Lilly, other from Samsung, other from BMS, other from Puma, other from MSD, grants from Immunomedics, grants and other from AstraZeneca, grants and other from Pfizer, other from Pierre Fabre and other from Merck outside the submitted work; In addition, SL has a patent EP14153692.0 pending. CD declares personal fees from Roche, Amgen, Merck-Serono, Bayer, Servier, Sanofi and research Grants from BMS. SL declares honoraria: Novartis, Roche, consulting: MSD oncology, Daiitchi Sankyo, molecular health, ownership nterest: Myriad, Reserche funding: Myriad genetics. Intellectual property: VMScope digital pathology software Recipient, Patent application: EP18209672 - cancer immunotherapy, Patent application EP20150702464 - therapy response, Patent application EP20150702464-therapy response. The other authors have no conflicts of interest to declare.

Publisher's note Springer Nature remains neutral with regard to jurisdictional claims in published maps and institutional affiliations.

Open Access This article is licensed under a Creative Commons Attribution 4.0 International License, which permits use, sharing, adaptation, distribution and reproduction in any medium or format, as long as you give appropriate credit to the original author(s) and the source, provide a link to the Creative Commons license, and indicate if changes were made. The images or other third party material in this article are included in the article's Creative Commons license, unless indicated otherwise in a credit line to the material. If material is not included in the article's Creative Commons license and your intended use is not permitted by statutory regulation or exceeds the permitted use, you will need to obtain permission directly from the copyright holder. To view a copy of this license, visit http://creativecommons. org/licenses/by/4.0/.

\section{References}

1. Denkert C, von Minckwitz G, Darb-Esfahani S, Lederer B, Heppner BI, Weber KE, et al. Tumour-infiltrating lymphocytes and prognosis in different subtypes of breast cancer: a pooled analysis of 3771 patients treated with neoadjuvant therapy. Lancet Oncol. 2018;19:40-50.

2. Hendry S, Salgado R, Gevaert T, Russell PA, John T, Thapa B, et al. Assessing tumor-infiltrating lymphocytes in solid tumors: a practical review for pathologists and proposal for a standardized method from the International Immunooncology Biomarkers Working Group: Part 1: assessing the host immune response, tils in invasive breast carcinoma and ductal carcinoma in situ, metastatic tumor deposits and areas for further research. Adv Anat Pathol. 2017;24:235-251.

3. Hendry S, Salgado R, Gevaert T, Russell PA, John T, Thapa B, et al. Assessing tumor-infiltrating lymphocytes in solid tumors: a practical review for pathologists and proposal for a standardized method from the International Immuno-Oncology Biomarkers Working Group: Part 2: TILs in melanoma, gastrointestinal tract carcinomas, non-small cell lung carcinoma and mesothelioma, endometrial and ovarian carcinomas, squamous cell carcinoma of the head and neck, genitourinary carcinomas, and primary brain tumors. Adv Anat Pathol. 2017;24:311-335.

4. Schmid P, Rugo HS, Adams S, Schneeweiss A, Barrios CH, Iwata $\mathrm{H}$, et al. Atezolizumab plus nab-paclitaxel as first-line treatment for unresectable, locally advanced or metastatic triple-negative breast cancer (IMpassion130): updated efficacy results from a randomised, double-blind, placebo-controlled, phase 3 trial. Lancet Oncol. 2020;21:44-59.

5. Perales-Puchalt A, Perez-Sanz J, Payne KK, Svoronos N, Allegrezza MJ, Chaurio RA, et al. Frontline science: microbiota reconstitution restores intestinal integrity after cisplatin therapy. $\mathrm{J}$ Leukoc Biol. 2018;103:799-805. 
6. Rutkowski MR, Stephen TL, Svoronos N, Allegrezza MJ, Tesone AJ, Perales-Puchalt A, et al. Microbially driven TLR5-dependent signaling governs distal malignant progression through tumorpromoting inflammation. Cancer Cell. 2015;27:27-40.

7. Iida N, Dzutsev A, Stewart CA, Smith L, Bouladoux N, Weingarten RA, et al. Commensal bacteria control cancer response to therapy by modulating the tumor microenvironment. Science. 2013;342:967-970.

8. Viaud S, Saccheri F, Mignot G, Yamazaki T, Daillère R, Hannani $\mathrm{D}$, et al. The intestinal microbiota modulates the anticancer immune effects of cyclophosphamide. Science. 2013;342:971-976.

9. Sivan A, Corrales L, Hubert N, Williams JB, Aquino-Michaels $\mathrm{K}$, Earley ZM, et al. Commensal Bifidobacterium promotes antitumor immunity and facilitates anti-PD-L1 efficacy. Science. 2015;350:1084-1089.

10. Vétizou M, Pitt JM, Daillère R, Lepage P, Waldschmitt N, Flament $\mathrm{C}$, et al. Anticancer immunotherapy by CTLA-4 blockade relies on the gut microbiota. Science. 2015;350:1079-1084.

11. Routy B, Le Chatelier E, Derosa L, Duong CPM, Alou MT, Daillère R, et al. Gut microbiome influences efficacy of PD-1based immunotherapy against epithelial tumors. Science. 2018;359:91-97.

12. Matson V, Fessler J, Bao R, Chongsuwat T, Zha Y, Alegre M-L, et al. The commensal microbiome is associated with anti-PD-1 efficacy in metastatic melanoma patients. Science. 2018;359:104-108.

13. Zitvogel L, Ma Y, Raoult D, Kroemer G, Gajewski TF. The microbiome in cancer immunotherapy: diagnostic tools and therapeutic strategies. Science. 2018;359:1366-1370.

14. Gopalakrishnan V, Spencer CN, Nezi L, Reuben A, Andrews MC, Karpinets TV, et al. Gut microbiome modulates response to anti-PD-1 immunotherapy in melanoma patients. Science. 2018;359:97-103.

15. Mohiuddin JJ, Chu B, Facciabene A, Poirier K, Wang X, Doucette A, et al. Association of antibiotic exposure with survival and toxicity in patients with melanoma receiving immunotherapy. $J$ Natl Cancer Inst. 2020. https://doi.org/10.1093/jnci/djaa057.

16. Huang X-Z, Gao P, Song Y-X, Xu Y, Sun J-X, Chen X-W, et al. Antibiotic use and the efficacy of immune checkpoint inhibitors in cancer patients: a pooled analysis of 2740 cancer patients. Oncoimmunology. 2019;8:e1665973.

17. Lurienne L, Cervesi J, Duhalde L, de Gunzburg J, Andremont A, Zalcman G, et al. NSCLC immunotherapy efficacy and antibiotic use: a systematic review and meta-analysis. $\mathrm{J}$ Thorac Oncol. 2020;15:1147-1159.

18. Elkrief A, Derosa L, Kroemer G, Zitvogel L, Routy B. The negative impact of antibiotics on outcomes in cancer patients treated with immunotherapy: a new independent prognostic factor? Ann Oncol. 2019;30:1572-1579.

19. Derosa L, Routy B, Fidelle M, Iebba V, Alla L, Pasolli E et al. Gut bacteria composition drives primary resistance to cancer immunotherapy in renal cell carcinoma patients. Eur Urol. 2020. https:// doi.org/10.1016/j.eururo.2020.04.044.

20. Chen KLA, Liu X, Zhao YC, Hieronymi K, Rossi G, Auvil LS, et al. Long-term administration of conjugated estrogen and bazedoxifene decreased murine fecal $\beta$-glucuronidase activity without impacting overall microbiome community. Sci Rep. 2018;8:8166

21. Goedert JJ, Hua X, Bielecka A, Okayasu I, Milne GL, Jones GS, et al. Postmenopausal breast cancer and oestrogen associations with the IgA-coated and IgA-noncoated faecal microbiota. Br J Cancer. 2018;118:471-479.

22. Parida S, Sharma D. Microbial alterations and risk factors of breast cancer: connections and mechanistic insights. Cells. 2020;9. https://doi.org/10.3390/cells9051091.

23. Buqué A, Bloy N, Perez-Lanzón M, Iribarren K, Humeau J, Pol JG. et al. Immunoprophylactic and immunotherapeutic control of hormone receptor-positive breast cancer. Nat Commun 11:3819:2020. https://doi.org/10.1038/s41467-020-17644-0.

24. Caffa I, Spagnolo V, Vernieri C, Valdemarin F, Becherini P, Wei $\mathrm{M}$, et al. Fasting-mimicking diet and hormone therapy induce breast cancer regression. Nature. 2020;583:620-624.

25. Paul B, Royston KJ, Li Y, Stoll ML, Skibola CF, Wilson LS, et al. Impact of genistein on the gut microbiome of humanized mice and its role in breast tumor inhibition. PLoS ONE. 2017;12:e0189756.

26. Franzosa EA, McIver LJ, Rahnavard G, Thompson LR, Schirmer $\mathrm{M}$, Weingart G, et al. Species-level functional profiling of metagenomes and metatranscriptomes. Nat Methods. 2018;15:962-968.

27. Routy B, Gopalakrishnan V, Daillère R, Zitvogel L, Wargo JA, Kroemer G. The gut microbiota influences anticancer immunosurveillance and general health. Nat Rev Clin Oncol. 2018;15:382-396.

28. Roberti MP, Yonekura S, Duong CPM, Picard M, Ferrere G, Tidjani Alou M, et al. Chemotherapy-induced ileal crypt apoptosis and the ileal microbiome shape immunosurveillance and prognosis of proximal colon cancer. Nat Med. 2020;26:919-931.

29. Hakozaki T, Richard C, Okuma Y, Derosa L, Elkrief A, Zitvogel $\mathrm{L}$, et al. Gut microbiome to predict efficacy and immune-related toxicities in patients with advanced non-small cell lung cancer treated with anti-PD-1/PD-L1 antibody-based immunotherapy. JCO. 2020;38:3095-3095.

30. Zhu J, Liao M, Yao Z, Liang W, Li Q, Liu J, et al. Breast cancer in postmenopausal women is associated with an altered gut metagenome. Microbiome. 2018;6:136.

31. Desmedt C, Fornili M, Clatot F, Demicheli R, De Bortoli D, Di Leo A, et al. Differential benefit of adjuvant docetaxel-based chemotherapy in patients with early breast cancer according to baseline body mass index. JCO. 2020, JCO.19.01771.

32. Guan X, Ma F, Sun X, Li C, Li L, Liang F, et al. Gut microbiota profiling in patients with HER2-negative metastatic breast cancer receiving metronomic chemotherapy of capecitabine compared to those under conventional dosage. Front Oncol. 2020;10:902.

33. Fernández MF, Reina-Pérez I, Astorga JM, Rodríguez-Carrillo A, Plaza-Díaz J, Fontana L. Breast cancer and its relationship with the microbiota. Int J Environ Res Public Health. 2018; 15. https:// doi.org/10.3390/ijerph15081747.

34. Luu TH, Michel C, Bard J-M, Dravet F, Nazih H, BobinDubigeon C. Intestinal proportion of blautia sp. is associated with clinical stage and histoprognostic grade in patients with earlystage breast cancer. Nutr Cancer. 2017;69:267-275.

35. Buchta Rosean C, Bostic RR, Ferey JCM, Feng T-Y, Azar FN, Tung KS, et al. Preexisting commensal dysbiosis is a host-intrinsic regulator of tissue inflammation and tumor cell dissemination in hormone receptor-positive breast cancer. Cancer Res. 2019;79:3662-3675.

36. Dias RB, Rombo DM, Ribeiro JA, Henley JM, Sebastião AM. Adenosine: setting the stage for plasticity. Trends Neurosci. 2013;36:248-257.

37. Antonioli L, Csóka B, Fornai M, Colucci R, Kókai E, Blandizzi C, et al. Adenosine and inflammation: what's new on the horizon? Drug Discov Today. 2014;19:1051-1068.

38. Carman AJ, Mills JH, Krenz A, Kim D-G, Bynoe MS. Adenosine receptor signaling modulates permeability of the blood-brain barrier. J Neurosci. 2011;31:13272-13280.

39. Lee C-C, Chang C-P, Lin C-J, Lai H-L, Kao Y-H, Cheng S-J, et al. Adenosine augmentation evoked by an ENT1 inhibitor improves memory impairment and neuronal plasticity in the APP/ PS1 mouse model of Alzheimer's disease. Mol Neurobiol. 2018;55:8936-8952.

40. Williams-Karnesky RL, Sandau US, Lusardi TA, Lytle NK, Farrell JM, Pritchard EM, et al. Epigenetic changes induced by adenosine augmentation therapy prevent epileptogenesis. J Clin Invest. 2013;123:3552-3563. 
41. Cunha RA. How does adenosine control neuronal dysfunction and neurodegeneration? J Neurochem. 2016;139:1019-1055.

42. Chen J-F, Lee C, Chern Y. Adenosine receptor neurobiology: overview. Int Rev Neurobiol. 2014;119:1-49.

43. Grajeda-Iglesias C, Durand S, Daillère R, Iribarren K, Lemaitre F, Derosa L et al. Oral administration of Akkermansia muciniphila elevates systemic antiaging and anticancer metabolites. Aging. 2021; 13. https://doi.org/10.18632/aging.202739.

44. Ma L, Ni Y, Wang Z, Tu W, Ni L, Zhuge F, et al. Spermidine improves gut barrier integrity and gut microbiota function in dietinduced obese mice. Gut Microbes. 2020;12:1-19.

45. Lévesque S, Le Naour J, Pietrocola F, Paillet J, Kremer M, Castoldi F, et al. A synergistic triad of chemotherapy, immune checkpoint inhibitors, and caloric restriction mimetics eradicates tumors in mice. Oncoimmunology. 2019;8:e1657375.

46. Ferrere G, Tidjani Alou M, Liu P, Goubet A-G, Fidelle M, Kepp $\mathrm{O}$, et al. Ketogenic diet and ketone bodies enhance the anticancer effects of PD1 blockade. JCI Insight. 2020. https://doi.org/10. 1172/jci.insight. 145207.

47. Tanoue T, Morita S, Plichta DR, Skelly AN, Suda W, Sugiura Y, et al. A defined commensal consortium elicits CD8 T cells and anti-cancer immunity. Nature. 2019;565:600-605.

48. Perraudeau F, McMurdie P, Bullard J, Cheng A, Cutcliffe C, Deo A, et al. Improvements to postprandial glucose control in subjects with type 2 diabetes: a multicenter, double blind, randomized placebo-controlled trial of a novel probiotic formulation. $B M J$ Open Diabetes Res Care. 2020;8. https://doi.org/10.1136/bmjdrc2020-001319.

49. Depommier C, Everard A, Druart C, Plovier H, Van Hul M, Vieira-Silva $S$, et al. Supplementation with Akkermansia muciniphila in overweight and obese human volunteers: a proof-ofconcept exploratory study. Nat Med. 2019;25:1096-1103.

50. Godon JJ, Zumstein E, Dabert P, Habouzit F, Moletta R. Molecular microbial diversity of an anaerobic digestor as determined by small-subunit rDNA sequence analysis. Appl Environ Microbiol. 1997;63:2802-2813.
51. Suau A, Bonnet R, Sutren M, Godon JJ, Gibson GR, Collins MD, et al. Direct analysis of genes encoding 16S rRNA from complex communities reveals many novel molecular species within the human gut. Appl Environ Microbiol. 1999;65:4799-4807.

52. Criscuolo A, Brisse S. AlienTrimmer: a tool to quickly and accurately trim off multiple short contaminant sequences from high-throughput sequencing reads. Genomics. 2013;102:500-506.

53. Beghini F, McIver LJ, Blanco-Míguez A, Dubois L, Asnicar F, Maharjan S, et al. Integrating taxonomic, functional, and strain-level profiling of diverse microbial communities with bioBakery 3 . Microbiology. 2020. https://doi.org/10.1101/2020.11.19.388223.

54. Li J, Jia H, Cai X, Zhong H, Feng Q, Sunagawa S, et al. An integrated catalog of reference genes in the human gut microbiome. Nat Biotechnol. 2014;32:834-841.

55. Langmead B, Salzberg SL. Fast gapped-read alignment with Bowtie 2. Nat Methods. 2012;9:357-359.

56. Cotillard A, Kennedy SP, Kong LC, Prifti E, Pons N, Le Chatelier E, et al. Dietary intervention impact on gut microbial gene richness. Nature. 2013;500:585-588.

57. Le Chatelier E, Nielsen T, Qin J, Prifti E, Hildebrand F, Falony G, et al. Richness of human gut microbiome correlates with metabolic markers. Nature. 2013;500:541-546.

58. Nielsen HB, Almeida M, Juncker AS, Rasmussen S, Li J, Sunagawa $\mathrm{S}$, et al. Identification and assembly of genomes and genetic elements in complex metagenomic samples without using reference genomes. Nat Biotechnol. 2014;32:822-828.

59. Segata N, Izard J, Waldron L, Gevers D, Miropolsky L, Garrett WS, et al. Metagenomic biomarker discovery and explanation. Genome Biol. 2011;12:R60.

60. Viltard M, Durand S, Pérez-Lanzón M, Aprahamian F, Lefevre D, Leroy $\mathrm{C}$, et al. The metabolomic signature of extreme longevity: naked mole rats versus mice. Aging. 2019;11:4783-4800.

61. Mattarollo SR, Loi S, Duret H, Ma Y, Zitvogel L, Smyth MJ. Pivotal role of innate and adaptive immunity in anthracycline chemotherapy of established tumors. Cancer Res. 2011;71:4809-4820.

\section{Affiliations}

\section{Safae Terrisse ${ }^{1,2,3,4} \cdot$ Lisa Derosa ${ }^{1,2,5} \cdot$ Valerio lebba $^{1,2} \cdot$ François Ghiringhelli $\mathbb{C}^{6,7,8,9} \cdot$ Ines Vaz-Luis ${ }^{10,11}$. Guido Kroemer $\mathbb{C}^{12,13,14,15} \cdot$ Marine Fidelle ${ }^{1,2,3,5} \cdot$ Stergios Christodoulidis $\mathbb{C}^{16,17} \cdot$ Nicola Segata $\mathbb{C}^{18,19}$. Andrew Maltez Thomas $\mathbb{1}^{19}$. Anne-Laure Martin ${ }^{20}$. Aude Sirven ${ }^{20} \cdot$ Sibille Everhard $^{20} \cdot$ Fanny Aprahamian $^{13}$. Nitharsshini Nirmalathasan ${ }^{13} \cdot$ Romy Aarnoutse $\mathbb{C}^{21,22,23} \cdot$ Marjolein Smidt ${ }^{21,22,23} \cdot$ Janine Ziemons $\mathbb{1}^{21,22,23}$. Carlos Caldas $\mathbb{1}^{24}$. Sibylle Loibl ${ }^{25,26}$. Carsten Denkert ${ }^{27}$. Sylvere Durand ${ }^{13} \cdot$ Claudia Iglesias $^{13}$. Filippo Pietrantonio ${ }^{28} \cdot$ Bertrand Routy $^{29} \cdot$ Fabrice André ${ }^{1,10,11,16} \cdot$ Edoardo Pasolli $^{30,31} \cdot$ Suzette Delaloge $e^{1,11}$. Laurence Zitvogel $\mathbb{D}^{1,2,3,5}$}

1 Gustave Roussy Cancer Center, Villejuif, France

2 INSERM U1015, Equipe Labellisée par la ligue Contre le Cancer, Villejuif, France

3 University Paris Saclay, School of Medicine, Le Kremlin-Bicêtre, France

4 Department of Medical Oncology, Saint Louis Hospital, Paris, France

5 Center of Clinical Investigations in Biotherapies of Cancer (CICBT), Villejuif, France

6 Research Platform in Biological Oncology, Dijon, France
7 GIMI Genetic and Immunology Medical Institute, Dijon, France

8 University of Burgundy-Franche Comté, Dijon, France

9 Department of Medical Oncology, Center GF Leclerc, Dijon, France

10 INSERM U 981, Gustave Roussy, Villejuif, Île-de-France, France

11 Department of Medicine, Breast Cancer committee, Gustave Roussy, Villejuif, France

12 INSERM U1138, Equipe Labelisée par la ligue Contre le Cancer, Centre de Recherche des Cordeliers, Paris, France 
13 Cell Biology and Metabolomics Platforms, Gustave Roussy Cancer Campus, Villejuif, France

Université de Paris, Paris, France

15 Sorbonne Université, Paris, France

16 University Paris Saclay, Saint-Aubain, France

17 Prism Precision Medicine Center, Gustave Roussy, Villejuif, France

18 Department of Medical Sciences, University of Trieste, Trieste, Italy

Department CIBIO, University of Trento, Trento, Italy

20 UNICANCER, Paris, France

21 Faculty of Health, Medicine \& Life Sciences, Department of Surgery, Maastricht, The Netherlands

22 GROW School for Oncology \& Developmental Biology, Maastricht, The Netherlands
23 Maastricht University, Maastricht, The Netherlands

24 Cancer Research UK Cambridge Institute, University of Cambridge, Li Ka Shing Centre, Robinson Way, Cambridge, UK

25 Goethe University Frankfurt, Frankfurt, Germany

26 Clinical Consultant Centre for Haematology and Oncology, Frankfurt, Germany

27 Philipps-University Marburg and University Hospital Marburg (UKGM), Marburg, Germany

28 Fondazione IRCCS Istituto Nazionale dei Tumori, Milano, Italy

29 Division d'hémato-oncologie, Département de Médicine, Centre Hospitalier de l'université de Montréal (CHUM), Montréal, Québec, Canada

30 Department of Agricultural Sciences, University of Naples Federico II, Portici, Italy

31 Task Force on Microbiome Studies, University of Naples Federico II, Naples, Italy 